\title{
Performance evaluation of variable transmission rate OFDM systems via network source coding
}

\author{
Te-Lung Kung* and Keshab K Parhi
}

\begin{abstract}
Studies related to the network source coding have addressed rate-distortion analysis in both noiseless and noisy channels. However, to the best of authors' knowledge, no prior work has studied network source coding in the context of orthogonal frequency division multiplexing (OFDM) systems. In addition, the system performance using network source coding schemes also remains unknown. In this article, we first propose two variable transmission rate (VTR) OFDM systems by utilizing network source coding schemes, and then evaluate the system performance of these two proposed VTR-OFDM systems. For the proposed VTR single-input single-output OFDM system, we employ the concept of a network with intermediate nodes to develop a 3-stage encoder/decoder, and the proposed encoder provides three different coding rates from 0.5 to 0.8 . As for the proposed VTR multi-band OFDM system, two correlated sources are simultaneously transmitted using the multiterminal source coding schemes, and two sources are encoded by different coding rates from 0.25 to 0.5 . Finally, compared with the traditional uncoded OFDM system, the proposed VTR-OFDM systems have at least 1 to $4 \mathrm{~dB}$ gain in signal-to-noise ratio to achieve the same symbol error rate in the additive white Gaussian noise channel.
\end{abstract}

Keywords: Network source coding, Single-input single-output OFDM, Multiterminal source coding, Multi-band OFDM, Variable transmission rate

\section{Introduction}

The rapid growth of communications systems for video, voice, and cellular telephone justifies great demands for mobile multimedia [1,2]. These multimedia services will require high data rate transmissions over broadband radio channels. Due to the limited spectral resource, it is impractical to increase the bandwidth for data transmission. In the case of wired networks, high data rate services are not a problem due to the channel characteristic; however, high data rate transmissions lead to an additional technical consideration for wireless communications networks. Moreover, the further development of the advanced network technologies is constrained by the amount of information that can be sent through the corresponding networks. In wired or wireless network, where

*Correspondence: kungx012@umn.edu

Department of Electrical and Computer Engineering, University of Minnesota, Minneapolis, MN, 55455, USA bandwidth is strictly limited, it is imperative to use efficient data representations or source codes for optimizing network system performance [3,4]. In order to transmit data stream over communications networks with limited bandwidth, network source coding can be used to solve this problem.

Network source coding expands the data compression problem for networks beyond the point-to-point network introduced by Shannon [5]. These include networks with multiple transmitters, multiple receivers, decoder side information, intermediate nodes, and any combination of these features. Thus, network source coding attempts to bridge the gap between point-to-point network and recent complicated network environments. Network source coding is a promising technique that provides many advantages, including source dependence, functional demands, and resource sharing [6-12]. By utilizing network source coding, the system performance can be improved by using correlated sources. This leads to an

\section{Springer}

(c) 2013 Kung and Parhi; licensee Springer. This is an Open Access article distributed under the terms of the Creative Commons Attribution License (http://creativecommons.org/licenses/by/2.0), which permits unrestricted use, distribution, and reproduction in any medium, provided the original work is properly cited. 
adjustable transmission rate, and the input data streams share the entire network resource during the transmission. If decoders have the information about the relation of transmitted data sources, the input data sources can be reconstructed without any loss in the noiseless channel.

High data rate wireless communications are limited not only by noise but also by the inter-symbol interference (ISI) from the dispersion of the wireless communications channel. Orthogonal frequency division multiplexing (OFDM) systems have been adopted in various wireless communications standards due to their high spectrum efficiency and robustness against the frequency selective fading channels $[1,13]$. In addition, OFDM is a prominent technique suitable for high data rate transmissions in multicarrier communications systems, because of the potential to either partially reduce or completely eliminate the adverse ISI effect. In OFDM systems, a wideband channel is converted to a set of narrowband subchannels, and the data is transmitted using all subchannels. Multi-band OFDM (MB-OFDM) systems group all subchannels into multiple subbands, and then transmit data in different subbands simultaneously $[14,15]$. Hence, the multi-band signal processing technique can be viewed as either a multiple access scheme that allocates subbands to transmit different users' data at the same time or an approach to employ the frequency diversities for data transmission in order to improve the system performance.

To the best of authors' knowledge, the following properties have not been demonstrated yet [6-12,16]: (1) the variable transmission rate (VTR) OFDM communications systems using network source coding, and (2) the performance improvement after applying the network source coding to OFDM systems. In this article, two VTR-OFDM communications systems are proposed to evaluate the performance of network source coding schemes. First, a VTR single-input single-output OFDM (VTR-SISO-OFDM) system is proposed. The proposed system generates different transmission rates using a proposed 3-stage encoder. Functions implemented in this 3-stage encoder determine the codebook sizes for storage and the achievable rates for data transmission. The proposed 3-stage encoder consists of three different mapping functions and their corresponding encoders using arithmetic coding scheme. The performance of the VTR-SISOOFDM system based on the proposed 3-stage encoder is evaluated. Then, a VTR multi-band OFDM (VTR-MBOFDM) system is proposed. The proposed VTR-MBOFDM system transmits two correlated sources using the multiterminal source coding scheme [7,17-19]. The transmission rates are adjusted based on four switch configurations at the transmitter, and 16 switch configurations control the information interchanges during the entire data transmission. The performance of the proposed VTR-MB-OFDM system for these 16 switch configurations is evaluated. We then analyze the variable transmission bit rate for these two proposed systems. The advantages of the proposed systems include availability of different transmission rates, abilities of different users to share the network resource, and robustness in wireless communications environments. Moreover, compared with the traditional uncoded OFDM systems, simulation results show the proposed systems obtain at least 1 to $4 \mathrm{~dB}$ gain in signal-to-noise ratio (SNR) to achieve the same symbol error rate (SER) in the additive white Gaussian noise (AWGN) channel. In addition, the rate factors of these two proposed systems vary from 0.55 to 0.96 .

The rest of the article is organized as follows. In Section 2, a VTR-SISO-OFDM communications system is proposed. A VTR-MB-OFDM system based on the multiterminal source coding scheme is proposed in Section 3. Performance analysis and simulation results are presented in Section 4. Finally, the article is concluded in Section 5.

\section{The VTR-SISO-OFDM system}

We propose a VTR-SISO-OFDM system with three different transmission rates in this section. The main goal of the proposed VTR-SISO-OFDM system is to design a variable transmission rate system and to recover the source data with the help of the side information. A 3-stage encoder is proposed in Section 2.1. In Section 2.2, the feasibilities of functions used in the encoder and the variable transmission rates of the proposed VTR-SISO-OFDM system are discussed. Finally, the corresponding decoder is described in Section 2.3.

\subsection{The 3-stage encoder of the VTR-SISO-OFDM system}

Figure 1a shows the 3-stage encoder of the proposed VTRSISO-OFDM system. Let $L$ symbols be transmitted. Let $\mathbf{a}^{T}, \mathbf{b}^{T}, \mathbf{c}^{T}$, and $\mathbf{d}^{T}$ be discrete random variables, where $\mathbf{a}^{T}$ is the source data, $\mathbf{b}^{T}, \mathbf{c}^{T}$, and $\mathbf{d}^{T}$ represent the side information, and $\mathbf{a}^{T}, \mathbf{b}^{T}, \mathbf{c}^{T}$, and $\mathbf{d}^{T}$ are independent. Consider the source data $\left\{a_{i}\right\}$ is independently selected from the set $\{1,2, \ldots, A\}$, where $\mathbf{a}^{T}=\left[a_{1}, a_{2}, \ldots, a_{L}\right]=\left\{a_{i}\right\}$, $i \in\{1,2, \ldots, L\}, i$ is the sample index, the probability of $\mathbf{a}^{T}$ is

$$
p\left(\mathbf{a}^{T}\right)=\prod_{i=1}^{L} p\left(a_{i}\right)
$$

and the cardinality of $\mathbf{a}^{T}$ is $A^{L}$. Moreover, the length of side information, $\mathbf{b}^{T}, \mathbf{c}^{T}$, and $\mathbf{d}^{T}$, is also $L$, where $\mathbf{b}^{T}=$ $\left[b_{1}, b_{2}, \ldots, b_{L}\right]=\left\{b_{i}\right\}, \mathbf{c}^{T}=\left[c_{1}, c_{2}, \ldots, c_{L}\right]=\left\{c_{i}\right\}, \mathbf{d}^{T}=$ 


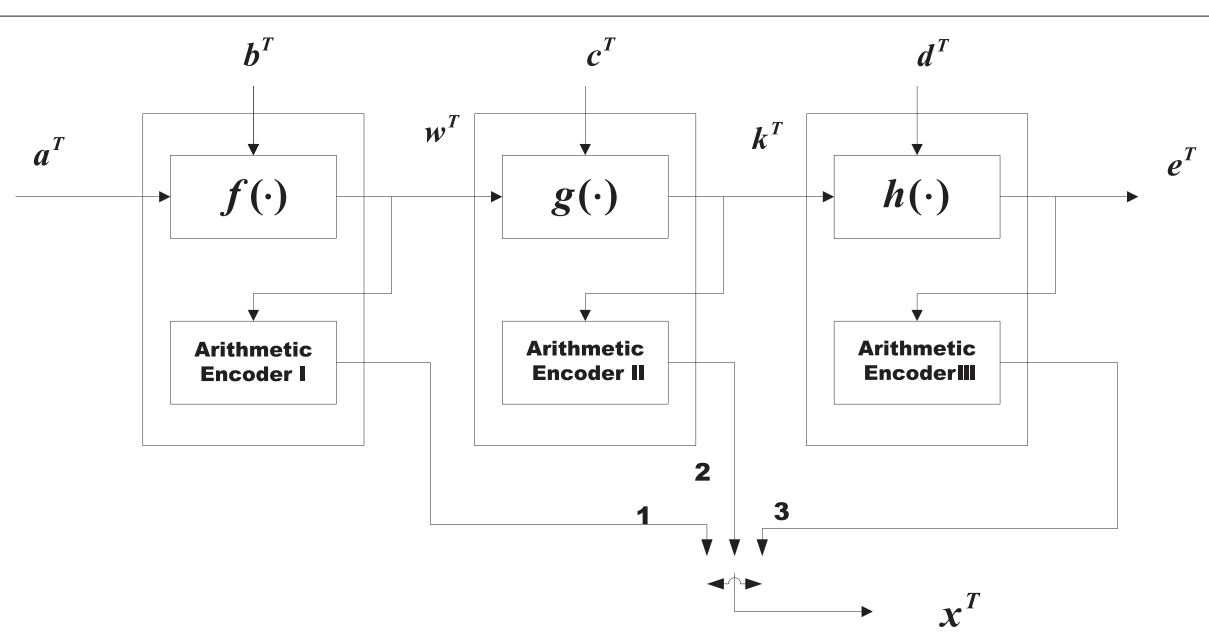

(a)

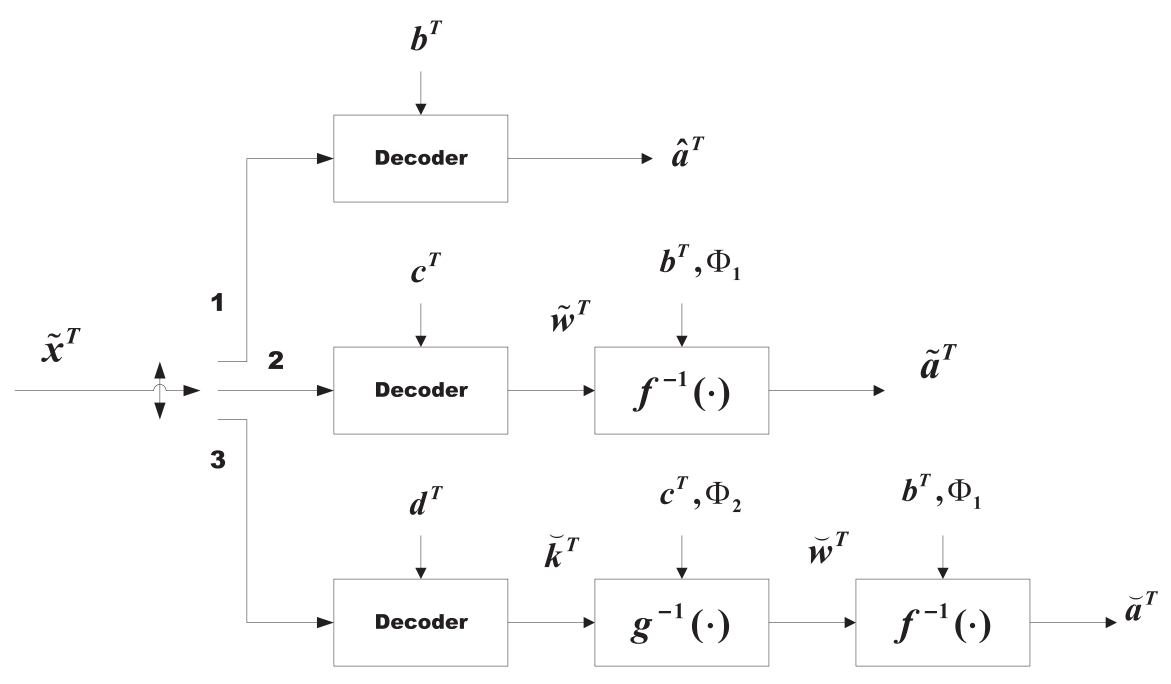

(b)

Figure 1 The 3-stage encoder/decoder of the proposed VTR-SISO-OFDM system. Figure 1 represents the proposed 3-stage (a) encoder/ (b) decoder in the proposed VTR-SISO-OFDM system. The proposed 3-stage encoder in Figure 1a consists of source data, mapping functions, and the side information. We utilize a specific switch to select different transmission rates. For the proposed 3-stage decoder in Figure $1 \mathrm{~b}$, the source data is reconstructed using reversible mapping functions and the side information.

$\left[d_{1}, d_{2}, \ldots, d_{L}\right]=\left\{d_{i}\right\}$, the probabilities of $\mathbf{b}^{T}, \mathbf{c}^{T}$, and $\mathbf{d}^{T}$ are given by:

$$
\begin{aligned}
p\left(\mathbf{b}^{T}\right) & =\prod_{i=1}^{L} p\left(b_{i}\right) \\
p\left(\mathbf{c}^{T}\right) & =\prod_{i=1}^{L} p\left(c_{i}\right) \\
p\left(\mathbf{d}^{T}\right) & =\prod_{i=1}^{L} p\left(d_{i}\right),
\end{aligned}
$$

and $\left\{b_{i}\right\},\left\{c_{i}\right\}$, and $\left\{d_{i}\right\}$ are randomly chosen from the sets $\{1,2, \ldots, B\},\{1,2, \ldots, C\}$, and $\{1,2, \ldots, D\}$, respectively. In
Figure 1a, the output symbols $\mathbf{w}^{T}=\left\{w_{i}\right\}, \mathbf{k}^{T}=\left\{k_{i}\right\}$, and $\mathbf{e}^{T}=\left\{e_{i}\right\}$ are

$$
\begin{aligned}
w_{i} & =f\left(a_{i}, b_{i}\right) \in\left\{\min \left(f\left(i_{1}, j_{1}\right)\right), \ldots, \max \left(f\left(i_{1}, j_{1}\right)\right)\right\}=\mathbf{m} \\
k_{i} & =g\left(w_{i}, c_{i}\right) \in\left\{\min \left(g\left(m_{i_{2}}, j_{2}\right)\right), \ldots, \max \left(g\left(m_{i_{2}}, j_{2}\right)\right)\right\}=\mathbf{y} \\
e_{i} & =h\left(k_{i}, d_{i}\right) \in\left\{\min \left(h\left(y_{i_{3}}, j_{3}\right)\right), \ldots, \max \left(h\left(y_{i_{3}}, j_{3}\right)\right)\right\}=\mathbf{o},
\end{aligned}
$$

where $f(\cdot), g(\cdot)$, and $h(\cdot)$ are injective or surjective functions, $i_{1} \in\{1,2, \ldots, A\}, j_{1} \in\{1,2, \ldots, B\}, i_{2} \in$ $\{1,2, \ldots, W\}, j_{2} \in\{1,2, \ldots, C\}, i_{3} \in\{1,2, \ldots, K\}, j_{3} \in$ $\{1,2, \ldots, D\}, \mathbf{m}, \mathbf{y}$, and $\mathbf{o}$ are ordered sets that contain distinct elements, and $W, K$, and $E$ are the number of 
elements in $\mathbf{m}, \mathbf{y}$, and $\mathbf{o}$, respectively. If $f(\cdot), g(\cdot)$, and $h(\cdot)$ are injective functions, the probabilities of $w_{i}, k_{i}$, and $e_{i}$ are given by:

$$
\begin{aligned}
& p\left(w_{i}\right)=p\left(f\left(a_{i}, b_{i}\right)\right)=p\left(a_{i}, b_{i}\right) \\
& p\left(k_{i}\right)=p\left(g\left(w_{i}, c_{i}\right)\right)=p\left(a_{i}, b_{i}, c_{i}\right) \\
& p\left(e_{i}\right)=p\left(h\left(k_{i}, d_{i}\right)\right)=p\left(a_{i}, b_{i}, c_{i}, d_{i}\right),
\end{aligned}
$$

and the corresponding entropies are

$$
\begin{aligned}
& H\left(w_{i}\right)=\sum_{i_{1}=1}^{A} \sum_{j_{1}=1}^{B}-p\left(f\left(i_{1}, j_{1}\right)\right) \cdot \log _{2}\left(p\left(f\left(i_{1}, j_{1}\right)\right)\right) \\
& H\left(k_{i}\right)=\sum_{i_{2}=1}^{W} \sum_{j_{2}=1}^{C}-p\left(g\left(m_{i_{2}}, j_{2}\right)\right) \cdot \log _{2}\left(p\left(g\left(m_{i_{2}}, j_{2}\right)\right)\right) \\
& H\left(e_{i}\right)=\sum_{i_{3}=1}^{K} \sum_{j_{3}=1}^{D}-p\left(h\left(y_{i_{3}}, j_{3}\right)\right) \cdot \log _{2}\left(p\left(h\left(y_{i_{3}}, j_{3}\right)\right)\right),
\end{aligned}
$$

where $p\left(f\left(i_{1}, j_{1}\right)\right)$ is the probability of the outcome $f\left(i_{1}, j_{1}\right)$, $p\left(a_{i}, b_{i}\right)$ is the joint probability of $\left(a_{i}, b_{i}\right), m_{i_{2}}$ means the $i_{2}$ th element in $\mathbf{m}$, and $y_{i_{3}}$ means the $i_{3}$ th element in $\mathbf{y}$. In addition, if $f(\cdot), g(\cdot)$, and $h(\cdot)$ are surjective functions, the probabilities of $w_{i}, k_{i}$, and $e_{i}$ are given by:

$$
\begin{aligned}
& p\left(w_{i}=i_{2}\right)=\sum_{i_{1}=1}^{A} p\left(i_{1}\right) \cdot\left\{\sum_{j_{1}=1}^{B} p\left(j_{1} \mid f\left(i_{1}, j_{1}\right)=i_{2}\right)\right\} \\
& p\left(k_{i}=i_{3}\right)=\sum_{i_{2}=1}^{W} p\left(i_{2}\right) \cdot\left\{\sum_{j_{2}=1}^{C} p\left(j_{2} \mid g\left(i_{2}, j_{2}\right)=i_{3}\right)\right\} \\
& p\left(e_{i}=i_{4}\right)=\sum_{i_{3}=1}^{K} p\left(i_{3}\right) \cdot\left\{\sum_{j_{3}=1}^{D} p\left(j_{3} \mid h\left(i_{3}, j_{3}\right)=i_{4}\right)\right\},
\end{aligned}
$$

and the corresponding entropies are

$$
\begin{aligned}
H\left(w_{i}\right)= & \sum_{i_{2}=1}^{W}-\left[\sum_{i_{1}=1}^{A} p\left(i_{1}\right) \cdot\left\{\sum_{j_{1}=1}^{B} p\left(j_{1} \mid f\left(i_{1}, j_{1}\right)=i_{2}\right)\right\}\right] \\
& \cdot \log _{2}\left(\sum_{i_{1}=1}^{A} p\left(i_{1}\right) \cdot\left\{\sum_{j_{1}=1}^{B} p\left(j_{1} \mid f\left(i_{1}, j_{1}\right)=i_{2}\right)\right\}\right.
\end{aligned}
$$

$$
\begin{aligned}
H\left(k_{i}\right)= & \sum_{i_{3}=1}^{K}-\left[\sum_{i_{2}=1}^{W} p\left(i_{2}\right) \cdot\left\{\sum_{j_{2}=1}^{C} p\left(j_{2} \mid g\left(i_{2}, j_{2}\right)=i_{3}\right)\right\}\right] \\
& \cdot \log _{2}\left(\sum_{i_{2}=1}^{W} p\left(i_{2}\right) \cdot\left\{\sum_{j_{2}=1}^{C} p\left(j_{2} \mid g\left(i_{2}, j_{2}\right)=i_{3}\right)\right\}\right. \\
H\left(e_{i}\right)= & \sum_{i_{4}=1}^{E}-\left[\sum_{i_{3}=1}^{K} p\left(i_{3}\right) \cdot\left\{\sum_{j_{3}=1}^{D} p\left(j_{3} \mid h\left(i_{3}, j_{3}\right)=i_{4}\right)\right\}\right] \\
& \cdot \log _{2}\left(\sum_{i_{3}=1}^{K} p\left(i_{3}\right) \cdot\left\{\sum_{j_{3}=1}^{D} p\left(j_{3} \mid h\left(i_{3}, j_{3}\right)=i_{4}\right)\right\},\right.
\end{aligned}
$$

where $i_{4} \in\{1,2, \ldots, E\}$ and $p\left(j_{1} \mid f\left(i_{1}, j_{1}\right)=i_{2}\right)$ is the conditional probability.

From Equation (4), if $f(\cdot), g(\cdot)$, and $h(\cdot)$ are injective functions, we do not need any extra information to reconstruct $a_{i}, w_{i}$, and $k_{i}$. However, from Equation (6), if $f(\cdot)$, $g(\cdot)$, and $h(\cdot)$ are surjective functions, the extra information is needed to create an one-to-one relationship between each input pair and the output symbol. Therefore, the actual input symbols $a_{i}, w_{i}$, and $k_{i}$ could be retrieved based on the extra information. For example, consider $f(\cdot)$ is a surjective function, where

$$
f\left(a_{i}, b_{i}\right)=\left|a_{i}-b_{i}\right| .
$$

Assume $A>4, B>4, L=3, \mathbf{a}^{T}=[1,3,2]$, and $\mathbf{b}^{T}=$ $[2,2,3]$. Then, we have

$$
\begin{aligned}
& \begin{array}{l}
f\left(a_{1}=1, b_{1}=2\right)=f\left(a_{2}=3, b_{2}=2\right) \\
\quad=f\left(a_{3}=2, b_{3}=3\right)=1
\end{array} \\
& \begin{array}{l}
f(1,2)=f(2,3)=|-1|=1 \\
f(3,2)=|1|=1
\end{array} \\
& \mathbf{w}^{T}=[1,1,1] .
\end{aligned}
$$

Without any extra information,

$$
\begin{aligned}
& a_{1}=f^{-1}\left(w_{1}, b_{1}\right)=f^{-1}(1,2)=\delta_{1} \\
& a_{2}=f^{-1}\left(w_{2}, b_{2}\right)=f^{-1}(1,2)=\delta_{1} \\
& a_{3}=f^{-1}\left(w_{3}, b_{3}\right)=f^{-1}(1,3)=\delta_{2},
\end{aligned}
$$

where $\delta_{1}$ could be 1 or 3 and $\delta_{2}$ could be 2 or 4 . Thus, $\mathbf{a}^{T}$ is given by:

$$
\begin{array}{r}
\mathbf{a}^{T} \in\{[1,1,2],[1,1,4],[1,3,2],[1,3,4] \\
[3,1,2],[3,1,4],[3,3,2],[3,3,4]\} .
\end{array}
$$

From Equation (9), in order to obtain the correct input symbol $a_{i}$, the extra information could be chosen by satisfying:

$$
\Phi_{1}=\left\{i \mid\left(a_{i}-b_{i}\right)<0, \forall i\right\},
$$


where $\Phi_{1}$ is a set and $\Phi_{1}$ contains sample index $i$ that satisfies $\left(a_{i}-b_{i}\right)<0$. Based on Equation (12), we have

$$
\Phi_{1}=\{1,3\} .
$$

Therefore, with the help of the extra information, the input symbol is reconstructed by

$$
\begin{aligned}
& a_{i}=f^{-1}\left(w_{i}, b_{i}, \Phi_{1}\right) \\
& a_{i}=b_{i}-w_{i}, i \in \Phi_{1} \\
& a_{i}=b_{i}+w_{i}, i \notin \Phi_{1} .
\end{aligned}
$$

Based on Equation (14), we have

$$
\begin{aligned}
& a_{1}=f^{-1}\left(1,2, i \in \Phi_{1}\right)=1 \\
& a_{2}=f^{-1}\left(1,2, i \notin \Phi_{1}\right)=3 \\
& a_{3}=f^{-1}\left(1,3, i \in \Phi_{1}\right)=2 \\
& \mathbf{a}^{T}=[1,3,2] .
\end{aligned}
$$

Moreover, if $g(\cdot)$ and $h(\cdot)$ are surjective functions, $\Phi_{2}$ and $\Phi_{3}$ are the corresponding extra information to obtain $w_{i}=g^{-1}\left(k_{i}, c_{i}, \Phi_{2}\right)$ and $k_{i}=h^{-1}\left(e_{i}, d_{i}, \Phi_{3}\right)$, respectively.

After $\mathbf{w}^{T}, \mathbf{k}^{T}$, and $\mathbf{e}^{T}$ are generated, we apply the arithmetic coding scheme to encode each symbol in these data streams. First, we consider $f(\cdot), g(\cdot)$, and $h(\cdot)$ are injective functions. Codebooks for different arithmetic encoders are $\mathbf{Q}_{t}$, where $t \in\{1,2,3\}$. In Equation (16), $\mathbf{Q}_{t}$ contains the information of all possible input source data, side information, probabilities for each possible outcome, and the corresponding binary codeword as follows:

$$
\begin{aligned}
& \mathbf{Q}_{1}\left(l_{1},:\right)=\left[\left(i_{1}, j_{1}\right), f\left(i_{1}, j_{1}\right), p\left(f\left(i_{1}, j_{1}\right)\right), \mathbf{x}_{f\left(i_{1}, j_{1}\right)}^{T}\right] \\
& \mathbf{Q}_{2}\left(l_{2},:\right)=\left[\left(m_{i_{2}}, j_{2}\right), g\left(m_{i_{2}}, j_{2}\right), p\left(g\left(m_{i_{2}}, j_{2}\right)\right), \mathbf{x}_{g\left(m_{i_{2}}, j_{2}\right)}^{T}\right] \\
& \mathbf{Q}_{3}\left(l_{3},:\right)=\left[\left(y_{i_{3}}, j_{3}\right), h\left(y_{i_{3}}, j_{3}\right), p\left(h\left(y_{i_{3}}, j_{3}\right)\right), \mathbf{x}_{h\left(y_{i_{3}}, j_{3}\right)}^{T}\right] \\
& l_{1}=\left(i_{1}-1\right) \times B+j_{1} \\
& l_{2}=\left(i_{2}-1\right) \times C+j_{2} \\
& l_{3}=\left(i_{3}-1\right) \times D+j_{3}
\end{aligned}
$$

where $l_{1}, l_{2}$, and $l_{3}$ are the row indices, $\mathbf{Q}_{1}\left(l_{1},:\right)$ means the $l_{1}$ th row in $\mathbf{Q}_{1}, \mathbf{x}_{f\left(i_{1}, j_{1}\right)}^{T}$ is the codeword corresponding to the outcome $f\left(i_{1}, j_{1}\right)$, and the numbers of possible outcomes in $\mathbf{Q}_{1}, \mathbf{Q}_{2}$, and $\mathbf{Q}_{3}$ are $A \times B, W \times C$, and $K \times D$. In addition, how to generate the encoded bit stream $\mathbf{x}^{T}$ depends on the selected switch, where

$$
\mathbf{x}^{T} \in\left\{\mathbf{x}_{f\left(\mathbf{a}^{T}, \mathbf{b}^{T}\right)}^{T}, \mathbf{x}_{g\left(\mathbf{w}^{T}, \mathbf{c}^{T}\right)}^{T}, \mathbf{x}_{h\left(\mathbf{k}^{T}, \mathbf{d}^{T}\right)}^{T}\right\}
$$

$f\left(\mathbf{a}^{T}, \mathbf{b}^{T}\right)=\left\{f\left(a_{i}, b_{i}\right), \forall i\right\}, g\left(\mathbf{w}^{T}, \mathbf{c}^{T}\right)=\left\{g\left(w_{i}, c_{i}\right), \forall i\right\}$, and $h\left(\mathbf{k}^{T}, \mathbf{d}^{T}\right)=\left\{h\left(k_{i}, d_{i}\right), \forall i\right\}$. From Equation (16), if we introduce more and more stages in the encoder, the Hamming distances between any two adjacent codewords at the output of higher stages become smaller and smaller.
The encoding rates $\left(R_{\text {enc }, S_{t}}\right)$ of the 3-stage encoder are

$$
\begin{aligned}
& R_{\mathrm{enc}, S_{1}}=\sum_{i_{1}=1}^{A} \sum_{j_{1}=1}^{B}\left[1+\left\lceil-\log _{2} p\left(i_{1}, j_{1}\right)\right\rceil\right] \cdot p\left(i_{1}, j_{1}\right) \\
& R_{\mathrm{enc}, S_{2}}=\sum_{i_{2}=1}^{W} \sum_{j_{2}=1}^{C}\left[1+\left\lceil-\log _{2} p\left(m_{i_{2}}, j_{2}\right)\right\rceil\right] \cdot p\left(m_{i_{2}}, j_{2}\right) \\
& R_{\mathrm{enc}, S_{3}}=\sum_{i_{3}=1}^{K} \sum_{j_{3}=1}^{D}\left[1+\left\lceil-\log _{2} p\left(y_{i_{3}}, j_{3}\right)\right\rceil\right] \cdot p\left(y_{i_{3}}, j_{3}\right) .
\end{aligned}
$$

Moreover, the 3-stage encoder provides three different lengths of $\mathbf{x}^{T}$, because the numbers of possible outcomes in $\mathbf{w}^{T}, \mathbf{k}^{T}$, and $\mathbf{e}^{T}$ are different. These three different lengths of $\mathbf{x}^{T}$ are

$$
\begin{aligned}
\operatorname{Len}_{S_{1}} & =\sum_{i=1}^{L} \operatorname{Len}\left(x_{f\left(a_{i}, b_{i}\right)}^{T}\right) \\
& =\sum_{i=1}^{L}\left[1+\left\lceil-\log _{2} p\left(a_{i}, b_{i}\right)\right\rceil\right] \\
\operatorname{Len}_{S_{2}} & =\sum_{i=1}^{L} \operatorname{Len}\left(x_{g\left(w_{i}, c_{i}\right)}^{T}\right) \\
& =\sum_{i=1}^{L}\left[1+\left\lceil-\log _{2} p\left(a_{i}, b_{i}, c_{i}\right)\right\rceil\right] \\
\operatorname{Len}_{S_{3}} & =\sum_{i=1}^{L} \operatorname{Len}\left(x_{h\left(k_{i}, d_{i}\right)}^{T}\right) \\
& =\sum_{i=1}^{L}\left[1+\left\lceil-\log _{2} p\left(a_{i}, b_{i}, c_{i}, d_{i}\right)\right\rceil\right] .
\end{aligned}
$$

If $f(\cdot), g(\cdot)$, and $h(\cdot)$ are surjective functions, the codebooks $\mathbf{Q}_{t}$, the encoding rates $\left(R_{\text {enc, } S_{t}}\right)$, and three lengths of $\mathbf{x}^{T}$ are different from Equations (16), (18), and (19). Therefore, we utilize the probabilities $p\left(w_{i}=i_{2}\right), p\left(k_{i}=i_{3}\right)$, and $p\left(e_{i}=i_{4}\right)$ in Equation (6) to derive the corresponding codebooks, encoding rates, and different lengths of the encoded bit streams for surjective functions. First, the codebooks $\mathbf{Q}_{t}$ are

$$
\begin{aligned}
& \mathbf{Q}_{1}\left(l_{1},:\right)=\left[\left(i_{1}, j_{1}\right), f\left(i_{1}, j_{1}\right), p\left(w_{i}=i_{2}\right), \mathbf{x}_{w_{i}}^{T}\right] \\
& \mathbf{Q}_{2}\left(l_{2},:\right)=\left[\left(m_{i_{2}}, j_{2}\right), g\left(m_{i_{2}}, j_{2}\right), p\left(k_{i}=i_{3}\right), \mathbf{x}_{k_{i}}^{T}\right] \\
& \mathbf{Q}_{3}\left(l_{3},:\right)=\left[\left(y_{i_{3}}, j_{3}\right), h\left(y_{i_{3}}, j_{3}\right), p\left(e_{i}=i_{4}\right), \mathbf{x}_{e_{i}}^{T}\right] \\
& l_{1}=\left(i_{1}-1\right) \times B+j_{1} \\
& l_{2}=\left(i_{2}-1\right) \times C+j_{2} \\
& l_{3}=\left(i_{3}-1\right) \times D+j_{3} .
\end{aligned}
$$


Then, the encoding rates $\left(R_{\mathrm{enc}, S_{t}}\right)$ are given by:

$$
\begin{aligned}
& R_{\mathrm{enc}, S_{1}}=\sum_{i_{2}=1}^{W}\left[1+\left\lceil-\log _{2} p\left(w_{i}=i_{2}\right)\right\rceil\right] \cdot p\left(w_{i}=i_{2}\right) \\
& R_{\mathrm{enc}, S_{2}}=\sum_{i_{3}=1}^{K}\left[1+\left\lceil-\log _{2} p\left(k_{i}=i_{3}\right)\right\rceil\right] \cdot p\left(k_{i}=i_{3}\right) \\
& R_{\mathrm{enc}, S_{3}}=\sum_{i_{4}=1}^{E}\left[1+\left\lceil-\log _{2} p\left(e_{i}=i_{4}\right)\right\rceil\right] \cdot p\left(e_{i}=i_{4}\right) .
\end{aligned}
$$

Finally, three different lengths of $\mathbf{x}^{T}$ are

$$
\begin{aligned}
\operatorname{Len}_{S_{1}} & =\sum_{i=1}^{L} \operatorname{Len}\left(x_{f\left(a_{i}, b_{i}\right)}^{T}\right) \\
& =\sum_{i=1}^{L}\left[1+\left\lceil-\log _{2} p\left(w_{i}=i_{2}\right)\right\rceil\right] \\
\operatorname{Len}_{S_{2}} & =\sum_{i=1}^{L} \operatorname{Len}\left(x_{g\left(w_{i}, c_{i}\right)}^{T}\right) \\
& =\sum_{i=1}^{L}\left[1+\left\lceil-\log _{2} p\left(k_{i}=i_{3}\right)\right\rceil\right] \\
\operatorname{Len}_{S_{3}} & =\sum_{i=1}^{L} \operatorname{Len}\left(x_{h\left(k_{i}, d_{i}\right)}^{T}\right) \\
& =\sum_{i=1}^{L}\left[1+\left\lceil-\log _{2} p\left(e_{i}=i_{4}\right)\right\rceil\right] .
\end{aligned}
$$

From Equations (19) and (22), the proposed 3-stage encoder generates three different transmission rates by selecting different output signals through varying the switch $S_{t}$.

In communications systems, two different ways utilized to transmit the data are continuous data transmission and packet data transmission. For the continuous data transmission such as voice, the transmission rate is controlled by the transport layer. In this case, the receiver knows the transmission rate during the entire data flow. As for the packet data transmission, each packet consists of the header and the data, and the information of the switch configuration can be hidden in the header of each packet. Therefore, in order to adjust the transmission rate without notifying the receiver, the transmitter first encodes the switch configuration and then includes the encoded bits in the header. The length of the header depends on the coding rate of the error correcting codes. In this article, we consider continuous data transmission.

After the entire encoding process, we pad the input data, $\mathbf{x}^{T}$, with a certain number of zeros $\left(N_{z}\right)$ to maintain the total number of bits $\left(N_{b}\right)$ equal to $M \times N_{T}$, where $N_{z}=$
$M \times N_{T}-$ Len $_{S_{t}}, M$ is the number of bits to represent a symbol, and $N_{T}$ is the number of total subcarriers. Then, the modified bit stream, $\left[\mathbf{x}^{T} 0_{1 \times N_{z}}\right.$, is transmitted using a SISO-OFDM system.

\subsection{The feasibilities of functions and variable transmission rate analysis}

Choosing the proper mapping functions in the encoder provides two advantages: (1) the improvement of the system performance and (2) the adjustability of the transmission rate. In order to reduce the size of codebook and to reconstruct the source data perfectly at the receiver, it is necessary to choose the mapping functions that generate smaller cardinalities in the encoder. Also, the help of the extra information provides us much more feasible ways to choose the mapping functions without any constraint.

The variable transmission bit rates $\left(R_{b, S_{t}}\right)$ of the proposed VTR-SISO-OFDM system are

$$
\begin{aligned}
R_{b, S_{t}} & =f_{s} \times M \times \frac{N_{u}}{N_{T}+N_{C P}} \\
N_{u} & =\left\lceil\frac{\text { Len }_{S_{t}}}{M}\right\rceil,
\end{aligned}
$$

where $f_{s}$ is the sampling rate, $N_{u}$ is the number of used subcarriers, $N_{C P}$ is the length of cyclic prefix, and $\lceil\cdot\rceil$ is the ceiling function. The rate factor, $\frac{N_{u}}{N_{T}}$, is derived from $\frac{R_{b, S_{t}}}{R_{b \text {,raw }}}$, where $R_{b \text {,raw }}$ is the raw transmission rate, $R_{b \text {,raw }}=$ $f_{s} \times M \times \frac{N_{T}}{N_{T}+N_{C P}}$, and $\frac{N_{u}}{N_{T}}<1$. From Equation (23), the transmission rate can be adjusted by varying the number of used subcarriers, $N_{u}$. Moreover, the probability of each possible outcome affects the number of used subcarriers. Thus, the smaller the probability of each possible outcome, the higher the transmission rate. In Figure 1a, if the maximal transmission rate is achieved from the output bit stream through the switch $S_{3}$, the probability of each possible outcome is $p\left(i_{1}, j_{1}, j_{2}, j_{3}\right)$, where $f(\cdot), g(\cdot)$, and $h(\cdot)$ are injective functions. Furthermore, the operation bandwidth of the proposed VTR-SISO-OFDM system depends on the switch $S_{t}$, and the variable operation bandwidths $\left(B W_{S_{t}}\right)$ for the proposed VTR-SISO-OFDM system are

$$
\begin{aligned}
B W_{S_{t}} & =N_{u} \times \Delta f \\
& =\left\lceil\frac{\text { Len }_{S_{t}}}{M}\right\rceil \times \frac{B W}{N_{T}},
\end{aligned}
$$

where $\Delta f$ is the subcarrier spacing, $\Delta f=\frac{B W}{N_{T}}$, and $B W$ is the original operation bandwidth.

\subsection{The 3-stage decoder of the VTR-SISO-OFDM system}

At the receiver, the received bit stream is reconstructed by the demodulation process in the SISO-OFDM system. Once the receiver obtains the estimated bit stream $\tilde{\mathbf{x}}^{T}$, the proposed decoder proceeds to process the data. 
Figure 1b shows the proposed 3-stage decoder in the VTR-SISO-OFDM system. First, the receiver utilizes the switch configuration assigned by the transport layer to determine the decoding route. For example, if $S_{3}=1$, the decoder closes the switch $S_{3}$, and proceeds to decode $\tilde{\mathbf{x}}^{T}$. The side information $\mathbf{d}^{T}$ helps the arithmetic decoder 3 to perform the first stage decoding process. In the first stage, the arithmetic decoder 3 decodes $\tilde{\mathbf{x}}^{T}$ based on the minimum Hamming distance rule with the aid of $\mathbf{Q}_{3}$ and $\mathbf{d}^{T}$. Then, $\check{\mathbf{w}}^{T}$ is generated through performing $g^{-1}(\cdot)$ on $\check{\mathbf{k}}^{T}$, $\mathbf{c}^{T}$, and $\Phi_{2}$. Finally, we obtain the estimated source data $\check{\mathbf{a}}^{T}$ after performing the inverse functions $f^{-1}(\cdot)$ on the estimated sequence $\check{\mathbf{w}}^{T}$, the side information $\mathbf{b}^{T}$, and the extra information $\Phi_{1}$ as shown in Equation (25).

$$
\begin{aligned}
\check{\mathbf{a}}^{T} & =f^{-1}\left(\check{\mathbf{w}}^{T}, \mathbf{b}^{T}, \Phi_{1}\right) \\
& =f^{-1}\left(g^{-1}\left(\check{\mathbf{k}}^{T}, \mathbf{c}^{T}, \Phi_{2}\right), \mathbf{b}^{T}, \Phi_{1}\right),
\end{aligned}
$$

where $f^{-1}\left(\check{\mathbf{w}}^{T}, \mathbf{b}^{T}, \Phi_{1}\right)=\left\{f^{-1}\left(\check{w}_{i}, b_{i}, \Phi_{1}\right), \forall i\right\}$ and $g^{-1}\left(\check{\mathbf{k}}^{T}, \mathbf{c}^{T}, \Phi_{2}\right)=\left\{g^{-1}\left(\check{k}_{i}, c_{i}, \Phi_{2}\right), \forall i\right\}$. The entire decoding procedure is listed in Algorithm 1. In Algorithm 1, Dist $\{\cdot\}$ is the Hamming distance and $\mathbf{s}(i: j)$ means from the $i$ th sample to the $j$ th sample in $\mathbf{s}$.

\section{The VTR-MB-OFDM system}

In this section, a VTR-MB-OFDM system is proposed using the multiterminal source coding scheme. The main goal of the proposed VTR-MB-OFDM system is to design a variable transmission rate system and to evaluate the system performance under different conditions.

\subsection{The proposed VTR-MB-OFDM system and transmission rate analysis}

The proposed VTR-MB-OFDM system is shown in Figures 2 and 3 . In Figure 2, correlated information sequences $\mathbf{a}^{T}$ and $\mathbf{b}^{T}$ are generated by repeated independent drawings of a pair of discrete random variables, where $\mathbf{a}^{T}=\left[a_{1}, a_{2}, \ldots, a_{L}\right]=\left\{a_{i}\right\}, \mathbf{b}^{T}=\left[b_{1}, b_{2}, \ldots, b_{L}\right]=$ $\left\{b_{i}\right\}, a_{i} \in\{1,2, \ldots, A\}, b_{i} \in\{1,2, \ldots, B\}, i \in\{1,2, \ldots, L\}$, and $L$ is the total number of drawings. In the proposed VTR-MB-OFDM system, $\hat{f}(\cdot)$ is an injective function. Thus, the entropies of $\hat{f}\left(a_{i}, S_{2} \cdot b_{i}\right)$ and $\hat{f}\left(S_{1} \cdot a_{i}, b_{i}\right)$ are

$$
\begin{aligned}
& H\left(\hat{f}\left(a_{i}, S_{2} \cdot b_{i}\right)\right) \\
& =\left\{\begin{array}{l}
\sum_{i_{1}=1}^{A}-p\left(i_{1}\right) \cdot \log _{2}\left(p\left(i_{1}\right)\right), \text { if } S_{2}=0, \\
\sum_{i_{1}=1}^{A} \sum_{j_{1}=1}^{B}-p\left(i_{1}, j_{1}\right) \cdot \log _{2}\left(p\left(i_{1}, j_{1}\right)\right), \text { if } S_{2}=1,
\end{array}\right. \\
& H\left(\hat{f}\left(S_{1} \cdot a_{i}, b_{i}\right)\right) \\
& =\left\{\begin{array}{l}
\sum_{j_{1}=1}^{B}-p\left(j_{1}\right) \cdot \log _{2}\left(p\left(j_{1}\right)\right), \text { if } S_{1}=0, \\
\sum_{i_{1}=1}^{A} \sum_{j_{1}=1}^{B}-p\left(i_{1}, j_{1}\right) \cdot \log _{2}\left(p\left(i_{1}, j_{1}\right)\right), \text { if } S_{1}=1 .
\end{array}\right.
\end{aligned}
$$

X-encoder encodes $\hat{f}\left(\mathbf{a}^{T}, S_{2} \cdot \mathbf{b}^{T}\right)$ to $\mathbf{x}_{\text {enc }}^{T}$ using the arithmetic coding scheme, where the function $\hat{f}(\cdot)$ maps each pair $\left(a_{i}, S_{2} \cdot b_{i}\right)$ in $\left(\mathbf{a}^{T}, S_{2} \cdot \mathbf{b}^{T}\right)$ to an unique value and the

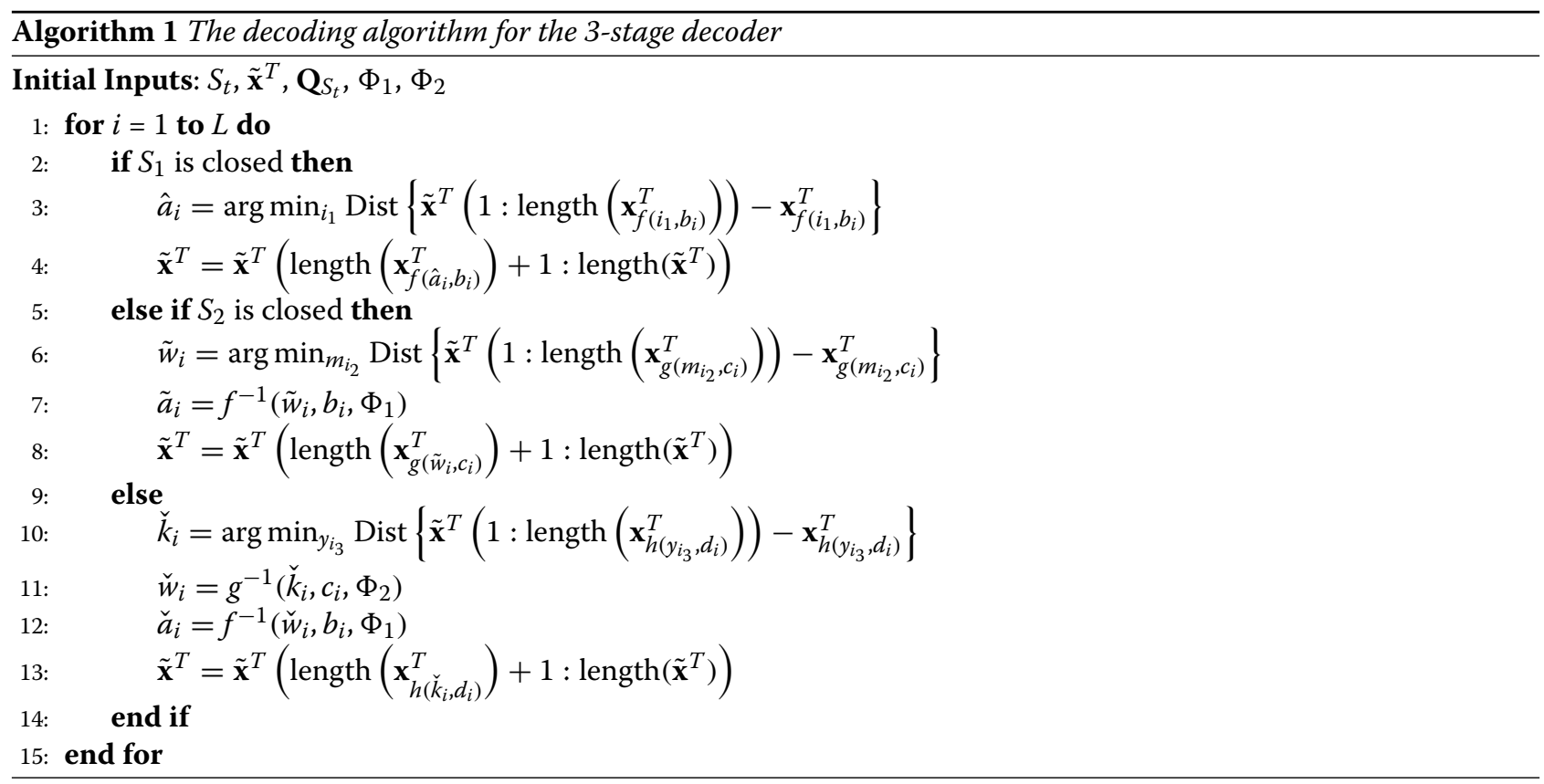




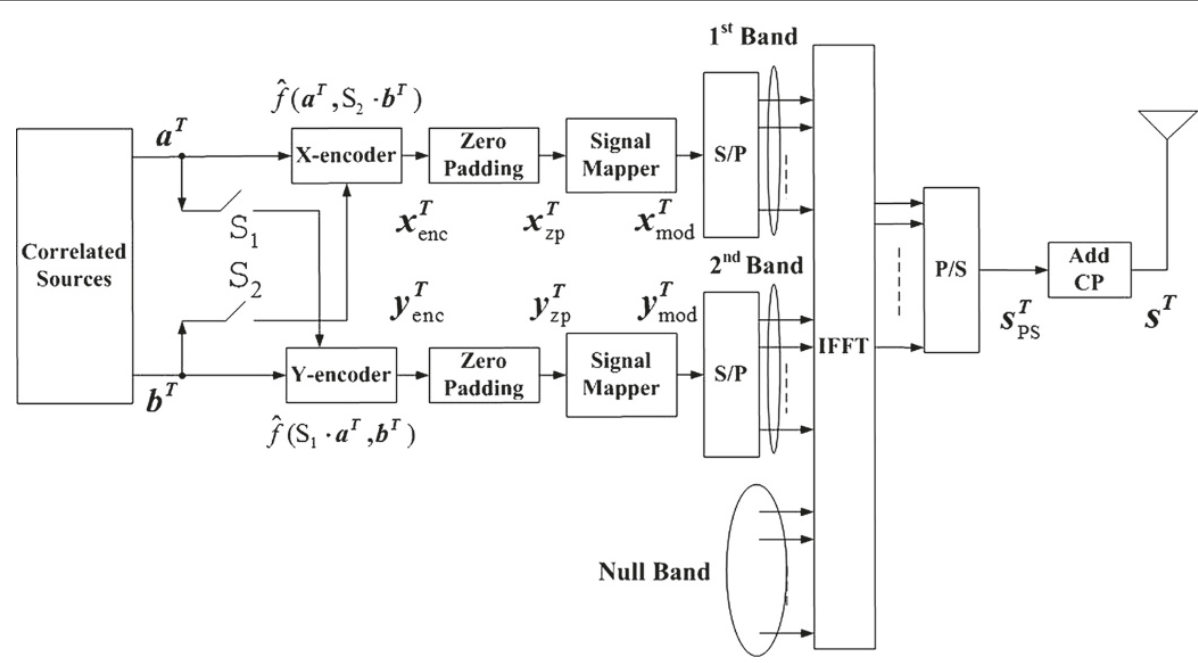

Figure 2 The transmitter in the proposed VTR-MB-OFDM communications system. Figure 2 represents the transmitter in the proposed VTR-MB-OFDM communications system. This proposed transmitter consists of a correlated source generator, a system architecture applying the multiterminal source coding, and the multi-band OFDM system.

length of $\mathbf{x}_{\text {enc }}^{T}$ depends on $S_{2}$. Codebooks, $\mathbf{Q}_{x}$ and $\mathbf{Q}_{y}$, used to generate $\mathbf{x}_{\mathrm{enc}}^{T}$ and $\mathbf{y}_{\mathrm{enc}}^{T}$ are

$$
\begin{aligned}
& \mathbf{Q}_{x}\left(\gamma_{1},:\right)=\left[\left(\alpha_{1}, \beta_{1}\right), \hat{f}\left(\alpha_{1}, \beta_{1}\right), p\left(\hat{f}\left(\alpha_{1}, \beta_{1}\right)\right), \mathbf{x}_{\hat{f}\left(\alpha_{1}, \beta_{1}\right)}^{T}\right] \\
& \mathbf{Q}_{y}\left(\gamma_{2},:\right)=\left[\left(\alpha_{2}, \beta_{2}\right), \hat{f}\left(\alpha_{2}, \beta_{2}\right), p\left(\hat{f}\left(\alpha_{2}, \beta_{2}\right)\right), \mathbf{y}_{\hat{f}\left(\alpha_{2}, \beta_{2}\right)}^{T}\right] \\
& \gamma_{1}=\beta_{1} \times A+\alpha_{1} \\
& \gamma_{2}=\alpha_{2} \times B+\beta_{2},
\end{aligned}
$$

where $\gamma_{1}$ and $\gamma_{2}$ are the row indices, $\alpha_{1} \in\{1,2, \ldots, A\}$, $\beta_{1} \in\{0,1, \ldots, B\}, \alpha_{2} \in\{0,1, \ldots, A\}, \beta_{2} \in\{1,2, \ldots, B\}$, $\mathbf{x}_{\hat{f}\left(\alpha_{1}, \beta_{1}\right)}^{T}$ and $\mathbf{y}_{\hat{f}\left(\alpha_{2}, \beta_{2}\right)}^{T}$ are the codewords corresponding to the possible outcomes $\hat{f}\left(\alpha_{1}, \beta_{1}\right)$ and $\hat{f}\left(\alpha_{2}, \beta_{2}\right)$, $\mathbf{x}_{\text {enc }}^{T}=$ $\mathbf{x}_{\hat{f}\left(\mathbf{a}^{T}, S_{2} \cdot \mathbf{b}^{T}\right)}^{T}$, and $\mathbf{y}_{\text {enc }}^{T}=\mathbf{y}_{\hat{f}\left(S_{1} \cdot \mathbf{a}^{T}, \mathbf{b}^{T}\right)}^{T}$. In order to satisfy the criterion of digital modulation, a zero vector is padded to the back of $\mathbf{x}_{\text {enc }}^{T}$ such that

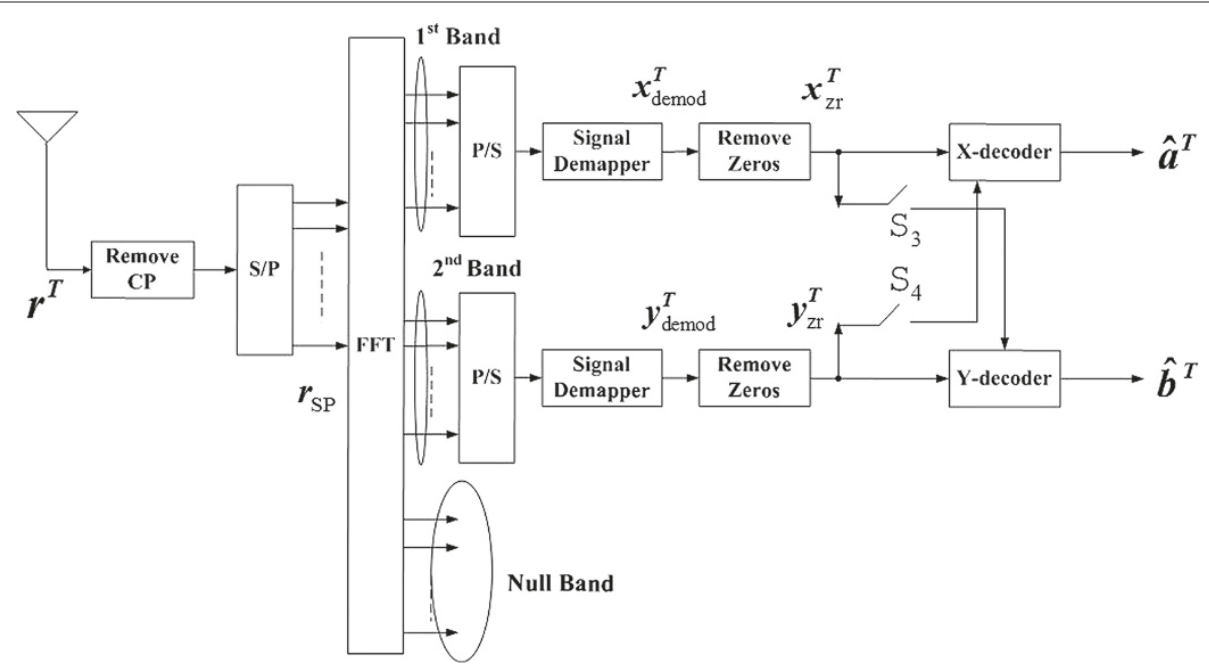

Figure 3 The receiver in the proposed VTR-MB-OFDM communications system. Figure 3 represents the receiver in the proposed VTR-MB-OFDM communications system. This proposed receiver consists of a decoding architecture of the multiterminal source coding and the multi-band OFDM system. 
where $X$ is the length of $\mathbf{x}_{\text {enc }}^{T}, M$ is the number of bits to represent a symbol, $\bmod ([M-\bmod (X, M)], M)$ is the length of the zero padding vector, the length of $\mathbf{x}_{z p}^{T}$ is $X+\bmod ([M-\bmod (X, M)], M), X$ is

$$
X=\left\{\begin{array}{l}
\sum_{i=1}^{L}\left[1+\left\lceil-\log _{2} p\left(a_{i}\right)\right\rceil\right], \text { if } S_{2}=0, \\
\sum_{i=1}^{L}\left[1+\left\lceil-\log _{2} p\left(a_{i}, b_{i}\right)\right\rceil\right], \text { if } S_{2}=1,
\end{array}\right.
$$

and the encoding rate $\left(R_{\mathrm{enc}, x\left[S_{2}\right]}\right)$ of X-encoder is

$$
\begin{aligned}
& R_{\text {enc }, x[0]}=\sum_{i_{1}=1}^{A}\left[1+\left\lceil-\log _{2} p\left(i_{1}\right)\right\rceil\right] \cdot p\left(i_{1}\right), \\
& R_{\text {enc }, x[1]}=\sum_{i_{1}=1}^{A} \sum_{j_{1}=1}^{B}\left[1+\left\lceil-\log _{2} p\left(i_{1}, j_{1}\right)\right\rceil\right] \cdot p\left(i_{1}, j_{1}\right) .
\end{aligned}
$$

After $\mathbf{x}_{z p}^{T}$ is modulated, we obtain $\mathbf{x}_{\text {mod }}^{T}$, and the bandwidth of the $1^{\text {st }}$ band occupied by $\mathbf{x}_{\text {mod }}^{T}$ is

$$
B W_{1^{s t}}=\left\lceil\frac{X}{M}\right\rceil \cdot \Delta f
$$

Y-encoder performs the same procedures to obtain $\mathbf{y}_{\text {enc }}^{T}$ by replacing $\hat{f}\left(\mathbf{a}^{T}, S_{2} \cdot \mathbf{b}^{T}\right)$ to $\hat{f}\left(S_{1} \cdot \mathbf{a}^{T}, \mathbf{b}^{T}\right)$. Then we pad a zero vector with length $\bmod ([M-\bmod (Y, M)], M)$ to the back of $\mathbf{y}_{\mathrm{enc}}^{T}$ in order to generate $\mathbf{y}_{z p}^{T}$. The length of $\mathbf{y}_{z p}^{T}$ is $Y+\bmod ([M-\bmod (Y, M)], M)$. In addition, the length of $\mathbf{y}_{\text {enc }}^{T}, Y$, is

$$
Y=\left\{\begin{array}{l}
\sum_{i=1}^{L}\left[1+\left\lceil-\log _{2} p\left(b_{i}\right)\right\rceil\right], \text { if } S_{1}=0, \\
\sum_{i=1}^{L}\left[1+\left\lceil-\log _{2} p\left(a_{i}, b_{i}\right)\right\rceil\right], \text { if } S_{1}=1,
\end{array}\right.
$$

and the encoding rate $\left(R_{\mathrm{enc}, y\left[S_{1}\right]}\right)$ of Y-encoder is

$$
\begin{aligned}
& R_{\text {enc }, y[0]}=\sum_{j_{1}=1}^{B}\left[1+\left\lceil-\log _{2} p\left(j_{1}\right)\right\rceil\right] \cdot p\left(j_{1}\right), \\
& R_{\text {enc }, y[1]}=\sum_{i_{1}=1}^{A} \sum_{j_{1}=1}^{B}\left[1+\left\lceil-\log _{2} p\left(i_{1}, j_{1}\right)\right\rceil\right] \cdot p\left(i_{1}, j_{1}\right) .
\end{aligned}
$$

Thus, the bandwidth of the $2^{\text {nd }}$ band is equal to

$$
B W_{2^{n d}}=\left\lceil\frac{Y}{M}\right\rceil \cdot \Delta f .
$$

From Equations (30) and (33), when $S_{1}$ and $S_{2}$ are equal to one, the lengths of two encoded bit streams, $X$ and $Y$, are identical; moreover, the allocated bandwidths of these two subbands, $B W_{1^{s t}}$ and $B W_{2^{n d}}$, are also the same.

Two serial-to-parallel (S/P) convertors are applied to $\mathbf{x}_{\text {mod }}^{T}$ and $\mathbf{y}_{\text {mod }}^{T}$ before the $N_{T}$-point inverse fast Fourier transform (IFFT) operation. Then, in order to perform a $N_{T}$-point IFFT operation, we insert a null band occupying $N_{\text {null }}$ subcarriers, where $N_{\text {null }}=N_{T}-\left\lceil\frac{X}{M}\right\rceil-\left\lceil\frac{Y}{M}\right\rceil$. After the $N_{T}$-point IFFT operation, $\mathbf{s}_{P S}^{T}$ is obtained using a parallel-to-serial $(\mathrm{P} / \mathrm{S})$ convertor. A cyclic prefix $(\mathrm{CP})$ with length $N_{C P}$ is inserted to the front of $\mathbf{s}_{P S}^{T}$ in order to reduce the ISI. Finally, $\mathbf{s}^{T}$ is transmitted using the radio frequency (RF) components, where

$$
\mathbf{s}^{T}=\left\{\begin{array}{l}
\mathbf{s}_{P S}^{T}\left(i+N_{T}-N_{C P}\right), i=0,1, \ldots, N_{C P}-1 \\
\mathbf{s}_{P S}^{T}(i), i=0,1, \ldots, N_{T}-1
\end{array} .\right.
$$

The transmission bit rate of the proposed VTR-MBOFDM system is related to the switches $S_{1}$ and $S_{2}$. Therefore, four different transmission bit rates $\left.\left(R_{b,\left[S_{1}\right.} S_{2}\right]\right)$ are

$$
\begin{aligned}
R_{b,[00]}= & \left\{\left\lceil\frac{\sum_{i=1}^{L}\left[1+\left\lceil-\log _{2} p\left(a_{i}\right)\right]\right]}{M}\right\rceil\right. \\
& \left.+\left\lceil\frac{\sum_{i=1}^{L}\left[1+\left\lceil-\log _{2} p\left(b_{i}\right)\right]\right]}{M}\right\rceil\right\} \times \frac{M \cdot f_{s}}{N_{T}+N_{C P}},
\end{aligned}
$$

$$
\begin{aligned}
\left.R_{b,[0} 1\right]= & \left\{\left\lceil\frac{\sum_{i=1}^{L}\left[1+\left\lceil-\log _{2} p\left(a_{i}\right)\right]\right]}{M}\right\rceil\right. \\
& \left.+\left\lceil\frac{\sum_{i=1}^{L}\left[1+\left\lceil-\log _{2} p\left(a_{i}, b_{i}\right)\right]\right]}{M}\right\rceil\right\} \times \frac{M \cdot f_{s}}{N_{T}+N_{C P}},
\end{aligned}
$$

$$
\begin{aligned}
R_{b,[10]}= & \left\{\left\lceil\frac{\sum_{i=1}^{L}\left[1+\left\lceil-\log _{2} p\left(a_{i}, b_{i}\right)\right\rceil\right]}{M}\right]\right. \\
& \left.+\left\lceil\frac{\sum_{i=1}^{L}\left[1+\left\lceil-\log _{2} p\left(b_{i}\right)\right]\right]}{M}\right]\right\} \times \frac{M \cdot f_{s}}{N_{T}+N_{C P}}, \\
R_{b,[11]}=\left\{\left\lceil\frac{\sum_{i=1}^{L}\left[1+\left\lceil-\log _{2} p\left(a_{i}, b_{i}\right)\right]\right]}{M}\right]\right. & \left.+\left\lceil\frac{\sum_{i=1}^{L}\left[1+\left\lceil-\log _{2} p\left(a_{i}, b_{i}\right)\right]\right]}{M}\right]\right\} \times \frac{M \cdot f_{s}}{N_{T}+N_{C P}} .
\end{aligned}
$$


Consider the data is transmitted over the AWGN channel. At the receiver as shown in Figure 3, the received signal $\mathbf{r}^{T}$ is

$$
\mathbf{r}^{T}=\mathbf{s}^{T}+\mathbf{n}^{T},
$$

where $\mathbf{n}^{T}$ is the AWGN noise. First, the receiver performs the cyclic prefix removal on $\mathbf{r}^{T}$, the S/P operation, and the $N_{T}$-point FFT operation on $\mathbf{r}_{S P}$. After the FFT operation, symbols allocated in the specific subbands are extracted, and then demodulated by the corresponding signal demapper. $\mathbf{x}_{\text {demod }}^{T}$ is generated using the information from the first $\left\lceil\frac{X}{M}\right\rceil$ subcarriers, and $\mathbf{y}_{\text {demod }}^{T}$ is also generated using the information from the $\left(\left\lceil\frac{X}{M}\right\rceil+1\right)$ th subcarrier to the $\left(\left\lceil\frac{X}{M}\right\rceil+\left\lceil\frac{Y}{M}\right\rceil\right)$ th subcarrier. Then, $\mathbf{x}_{z r}^{T}$ is obtained by removing $\bmod (M-\bmod (X, M), M)$ samples at the back of $\mathbf{x}_{\text {demod }}^{T}$, and $\mathbf{y}_{z r}^{T}$ is also obtained by removing $\bmod (M-\bmod (Y, M), M)$ samples at the back of $\mathbf{y}_{\text {demod }}^{T}$. Let us use $\mathbf{x}_{z r}^{T}$ to explain the decoding process, and $\mathbf{y}_{z r}^{T}$ is decoded using the same procedure. $\mathrm{X}$-decoder decodes $\mathbf{x}_{z r}^{T}$ by minimizing the Hamming distances between codewords in the codebook $\mathbf{Q}_{x}$ and the partial bit stream in $\mathbf{x}_{z r}^{T}$ with or without help of the side information through the switch $S_{3}$, and then the estimated source data $\hat{\mathbf{a}}^{T}$ is obtained. In Algorithm 2, we describe the overall decoding process used in X-decoder and Y-decoder.

\section{Performance analysis and simulation results}

We evaluate the performance of two proposed VTROFDM systems, including the VTR-SISO-OFDM system and the VTR-MB-OFDM system. Section 4.1 demonstrates the performance of the proposed VTR-SISOOFDM system, and then the performance of the proposed VTR-MB-OFDM system is demonstrated in Section 4.2.

\subsection{Performance evaluation of the VTR-SISO-OFDM system}

Assume 10000 packets are transmitted using the VTRSISO-OFDM system and each packet contains 2000 encoded messages. Consider the source data and the side information follow the same probability distribution $p(z)$,

$$
p(z=\lambda)=\frac{Z-\lambda+1}{\sum_{\lambda=1}^{Z}(Z-\lambda+1)},
$$

where $z$ is a random variable, $z \in\{1,2, \ldots, Z\}$, and $\sum_{\lambda=1}^{Z}(Z-\lambda+1)$ is the total number of possible outcomes.

The source data $\mathbf{a}^{T}=\left\{a_{i}\right\}$ is fed to the 3 -stage encoder in order to evaluate the system performance under three

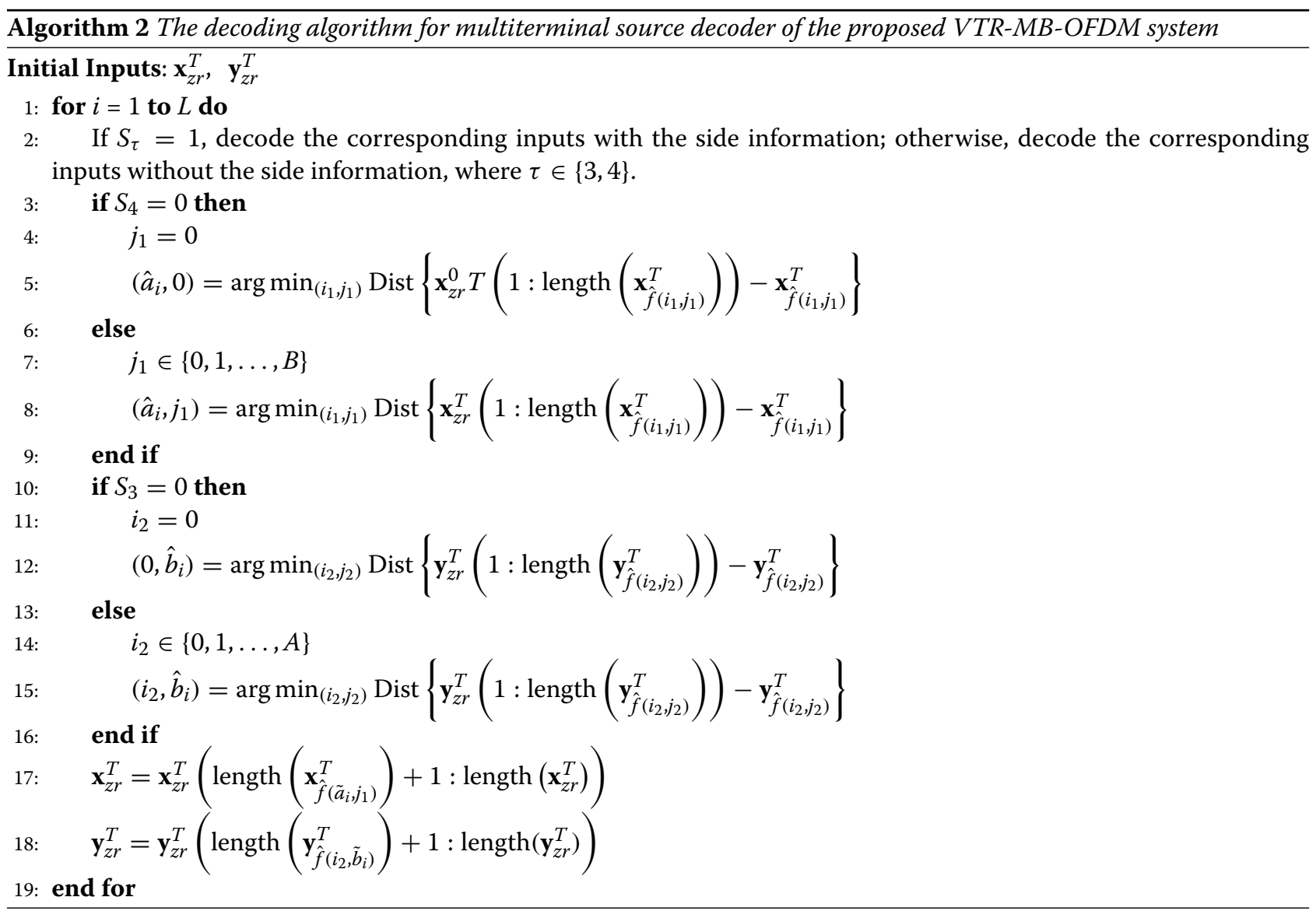


different transmission rates, where $L=2000$. Assume $A=100$, and the maximum values in the side information are given by $B=75, C=50$, and $D=25$ or $D=5$. The probability density function (pdf) of source data, $p\left(a_{i}\right)$, is

$$
p\left(a_{i}=i_{1}\right)=\frac{A-i_{1}+1}{N_{a}},
$$

where $N_{a}=\sum_{i_{1}=1}^{100}\left(100-i_{1}+1\right)=5050$. Then, the pdfs of the side information are

$$
\begin{aligned}
& p\left(b_{i}=j_{1}\right)=\frac{B-j_{1}+1}{N_{b}} \\
& p\left(c_{i}=j_{2}\right)=\frac{C-j_{2}+1}{N_{c}} \\
& p\left(d_{i}=j_{3}\right)=\frac{D-j_{3}+1}{N_{d}},
\end{aligned}
$$

where $N_{b}=\sum_{j_{1}=1}^{75}\left(75-j_{1}+1\right)=2850, N_{c}=\sum_{j_{2}=1}^{50}(50-$ $\left.j_{2}+1\right)=1275$, and $N_{d}=\sum_{j_{3}=1}^{D}\left(D-j_{3}+1\right)=325$ or 15 .

The mapping functions in the proposed 3-stage encoder, $f(\alpha, \beta), g(\alpha, \beta)$, and $h(\alpha, \beta)$, are

$$
\begin{aligned}
& f(\alpha, \beta)=\bmod (\alpha+\beta, \gamma) \\
& g(\alpha, \beta)=|\alpha-\beta| \\
& h(\alpha, \beta)=p(\alpha, \beta)
\end{aligned}
$$

where $\gamma$ is the maximum prime number in $A+B$ and $p(\alpha, \beta)$ is the joint probability function on $(\alpha, \beta)$. From Equation (41), $f(\cdot)$ and $g(\cdot)$ are surjective functions, and $h(\cdot)$ represents an injective function. After applying the mapping functions to the input symbols and the side information at each stage, the corresponding output symbols, $\mathbf{w}^{T}, \mathbf{k}^{T}$, and $\mathbf{e}^{T}$, are

$$
\begin{aligned}
w_{i} & =\bmod \left(a_{i}+b_{i}, 173\right) \\
k_{i} & =\left|w_{i}-c_{i}\right| \\
e_{i} & =p\left(k_{i}, d_{i}\right),
\end{aligned}
$$

where $\gamma=173$. Moreover, the cardinalities of $\mathbf{w}^{T}, \mathbf{k}^{T}$, and $\mathbf{e}^{T}$ are $W=173, K=172$, and $E=172 \times 25$ or $E=$ $172 \times 5$ depending on the value $D$. Thus, the transmission rate of the output signal at the $2^{\text {nd }}$ stage is the smallest, because $K$ is less than $W$ and $E$. In addition, $\Phi_{1}$ and $\Phi_{2}$ are chosen by satisfying:

$$
\begin{aligned}
& \Phi_{1}=\left\{i \mid\left(a_{i}+b_{i}\right)=175\right\} \\
& \Phi_{2}=\left\{i \mid\left(w_{i}-c_{i}\right)<0\right\} .
\end{aligned}
$$

Once the output symbols $\mathbf{w}^{T}, \mathbf{k}^{T}$, and $\mathbf{e}^{T}$ are generated, we apply arithmetic code to encode each symbol to the specific codeword using the corresponding codebook at each stage. Cardinalities of different codebooks $Q_{t}$ used to encode symbols at each stage are $A \times B, W \times C$, and $E$. Two bits for rate adjustment are added to the front of the output in order to construct the input bit stream. Then, we use a SISO-OFDM system with 16-QAM signal mapper/demapper, 8192-point IFFT/FFT $\left(N_{T}=8192\right)$, and
$N_{C P}=256$ to transmit the data over the AWGN channel. Assume the operating bandwidth of the communications system is $20 \mathrm{MHz}$, and each sample is generated by a sampling frequency $5 \mathrm{MHz}$. The raw transmission bit rate of this SISO-OFDM system is $19.4 \mathrm{Mbps}$. In addition, the subcarrier spacing, $\Delta f$, is about $2.441 \mathrm{MHz}$.

Some system parameters of the proposed VTR-SISOOFDM system are listed in Table 1 , where $S_{1}, S_{2}$, and $S_{3}$ indicate which route we choose. In Table 1 , we evaluate the entropies, encoding rates, used subcarriers, occupied bandwidth, and transmission rates. First, we notice that the encoding rates are greater than the entropies at each stage. It is important that the transmission of the proposed VTR-SISO-OFDM system is admissible under this condition. Furthermore, at the first two stages of the encoder, the entropies, $H\left(w_{i}\right)$ and $H\left(k_{i}\right)$, and the encoding rates, $R_{\text {enc, } S_{1}}$ and $R_{\text {enc, } S_{2}}$, are almost the same, because the cardinality of $\mathbf{w}^{T}$ is approximately equal to the cardinality of $\mathbf{k}^{T}$. Then, the subcarriers used on average and the transmission rates of the output signals at the first two stages are also approximately equal because of this reason. At the third stage, Table 1 also shows that we can obtain higher encoding rates and higher entropies simply by increasing $D$; however, we can also expect that the Hamming distances between each two adjacent codewords become smaller and smaller with increasing $D$. In addition, we estimate the used subcarriers, the occupied bandwidths, and the transmission rates at each stage using $L \cdot R_{\mathrm{enc}, S_{t}}$

\begin{tabular}{|c|c|c|c|c|}
\hline & \multirow[t]{2}{*}{$S_{1}$} & \multirow[t]{2}{*}{$S_{2}$} & \multicolumn{2}{|c|}{$S_{3}$} \\
\hline & & & $D=25$ & $D=5$ \\
\hline Entropy & 7.4346 & 7.3323 & 11.7240 & 9.4815 \\
\hline $\begin{array}{l}\text { Encoding rate } \\
\text { (bits/sample), } R_{\text {enc, } S_{t}}\end{array}$ & 9 & 8.8570 & 13.2461 & 11.0735 \\
\hline $\begin{array}{l}\text { Averaged number of used } \\
\text { subcarrier, }\left\lceil\frac{L \cdot R_{\text {enc,St }}}{M}\right\rceil\end{array}$ & 4501 & 4429 & 6624 & 5537 \\
\hline $\begin{array}{l}\text { Approximately allocated } \\
\text { bandwidth }(\mathrm{MHz} \text {.), } \\
{\left[\frac{L \cdot R_{\text {enc,St }}}{M}\right] \cdot \Delta f}\end{array}$ & 11 & 10.8 & 16.2 & 13.5 \\
\hline $\begin{array}{l}\text { Transmission bit } \\
\text { rate }(\mathrm{Mbps}), R_{b, S_{t}}= \\
\left\lceil\frac{L \cdot R_{\text {enc }, S_{t}}}{M}\right\rceil \cdot \frac{f_{s} \cdot M}{N_{T}+N_{C P}}\end{array}$ & 10.7 & 10.5 & 15.7 & 13.1 \\
\hline
\end{tabular}
instead of $\operatorname{Len}_{S_{t}}$, because the entire packet transmission is a stochastic process which means Len $_{S_{t}}$ is not a constant, and $R_{\text {enc, }} S_{t}$ provides the average codeword length of possible output data samples. Furthermore, the rate factors, $\left\lceil\frac{L \cdot R_{\text {enc, } S_{t}}}{M}\right\rceil \frac{1}{N_{T}}$, at different stages are approximately equal to $0.55,0.675$, and 0.8 .

We demonstrate the performance of the proposed VTRSISO-OFDM system in Figure 4. In Figure 4, the SERs of the proposed VTR-SISO-OFDM system at different

Table 1 System parameters of the proposed VTR-SISO-OFDM system 


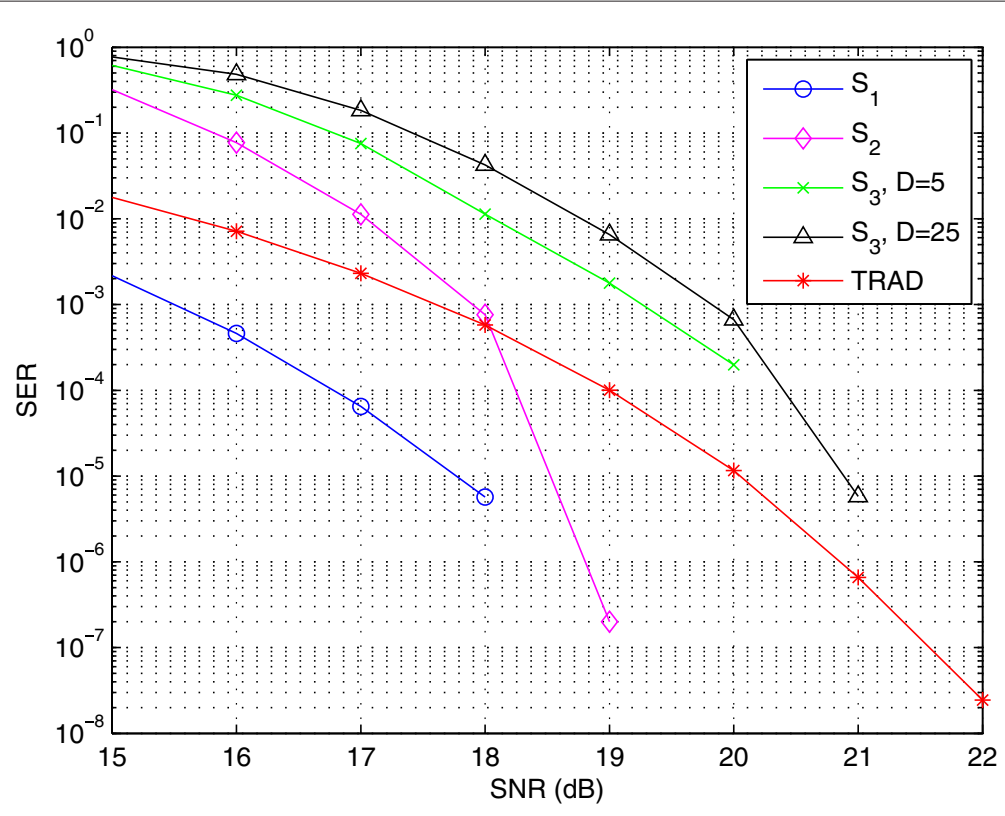

Figure 4 The performance of the proposed VTR-SISO-OFDM communications system at each stage with different $D$.

stages, $S_{1}, S_{2}$, and $S_{3}$, are compared with the performance of traditional uncoded OFDM system (TRAD). The SER of traditional uncoded OFDM system implies how many bits are incorrect. For example, under SNR = $19 \mathrm{~dB}$, there are almost four incorrect bits during each packet transmission. If we transmit the data stream using the route $S_{1}$, zero SER is achievable when SNR is greater than $19 \mathrm{~dB}$. Although we can transmit data stream with higher rate using the route $S_{3}$, there are approximate two incorrect symbols under $\mathrm{SNR}=19 \mathrm{~dB}$. Reasons why we have higher SER using the route $S_{3}$ are the smaller Hamming distances between each two adjacent codewords and the error propagation during the entire decoding process. By decreasing $D$, we obtain better performance with lower transmission rate as shown in Table 1 and Figure 4.

4.2 Performance evaluation of the VTR-MB-OFDM system Assume 10000 packets are transmitted using the proposed VTR-MB-OFDM system and each packet contains 2000 correlated information sequences, $\mathbf{a}^{T}$ and $\mathbf{b}^{T}$. After the correlated sequences are generated, we apply the multiterminal source coding to encode $\mathbf{a}^{T}$ and $\mathbf{b}^{T}$ with or without the side information from each other, and then these 2000 correlated sequences are transmitted using two different subbands in MB-OFDM system. Each subband is occupied by 1000 encoded messages. In addition, the bandwidths and the transmission rates of these two subbands are not necessarily the same.
Let $A$ and $B$ be equal to $N$, and then correlated information sequences $\mathbf{a}^{T}$ and $\mathbf{b}^{T}$ are generated with 1000 independent drawings from ( $\left.\begin{array}{ll}\mathbf{h} & \mathbf{l}\end{array}\right)$, where $\mathbf{h}^{T}$ and $\mathbf{l}^{T}$ are

$$
\begin{aligned}
\mathbf{h}^{T} & =[\underbrace{1, \ldots, 1}_{N}, \underbrace{2, \ldots, 2}_{N-1}, \ldots, \underbrace{N-1, N-1}_{2}, \underbrace{N}_{1}] \\
\mathbf{l}^{T} & =[\underbrace{N}_{1}, \underbrace{N-1, N-1}_{2}, \ldots, \underbrace{2, \ldots, 2}_{N-1}, \underbrace{1, \ldots, 1}_{N}],
\end{aligned}
$$

$N$ is a constant, $\underbrace{1, \ldots, 1}_{N}$ means the number of 1 s is $N, L=$ 1000 , and the correlation matrix of these two sequences $\mathbf{h}^{T}$ and $\mathbf{l}^{T}$ when $N=50$ is

$$
\mathbf{R}=\left(\begin{array}{cc}
1 & -0.9302 \\
-0.9302 & 1
\end{array}\right)
$$

From the cross-correlation coefficient $\mathbf{R}_{12}$ or $\mathbf{R}_{21}, \mathbf{h}^{T}$ is highly correlated with $\mathbf{1}^{T}$. Each input pair $\left(a_{i}, b_{i}\right)$ is obtained by independent drawing from the row of ( $\left.\begin{array}{ll}\mathbf{h} & \mathbf{l}\end{array}\right)$. The pdfs of $p\left(a_{i}\right)$ and $p\left(b_{i}\right)$ are

$$
\begin{aligned}
p\left(a_{i}=h_{i}\right) & =\frac{N-h_{i}+1}{N \cdot(N+1) / 2} \\
p\left(b_{i}=l_{i}\right) & =\frac{N-l_{i}+1}{N \cdot(N+1) / 2} .
\end{aligned}
$$


Moreover, the joint probability of $p\left(a_{i}, b_{i}\right)$ is

$$
p\left(a_{i}=h_{i}, b_{i}=l_{i}\right)=\frac{\operatorname{OCCUR}\left(h_{i}, l_{i}\right)}{N \cdot(N+1) / 2},
$$

where $\operatorname{OCCUR}(x)$ is the occurrence of $x$.

We evaluate the performance of the proposed VTR-MBOFDM system using two different mapping functions, and these two mapping functions are

$$
\begin{aligned}
& \hat{f}_{1}(\alpha, \beta)=p(\alpha, \beta) \\
& \hat{f}_{2}(\alpha, \beta)=\bmod (\alpha+\beta, \gamma),
\end{aligned}
$$

where $\gamma$ is the maximum prime number in $2 \cdot N$ and $p(\alpha, \beta)$ is the joint probability function on $(\alpha, \beta)$. Thus, $\hat{f}_{1}(\cdot)$ and $\hat{f}_{2}(\cdot)$ are injective functions. For the mapping function $\hat{f}_{1}(\cdot)$, we choose $N$ to be 10 , because we can achieve better performance using smaller $N$ from the simulation results in Section 4.1. Then, $A$ and $B$ are equal to 10 , and the number of distinct possible outcomes $\left(h_{i}, l_{i}\right)$ is 17 . After applying the mapping function $\hat{f}_{1}(\cdot)$ to the input pair $\left(a_{i}, b_{i}\right)$ with different information interchanges, the outputs are $p\left(a_{i}, S_{2} \cdot b_{i}\right)$ or $p\left(S_{1} \cdot a_{i}, b_{i}\right)$. As for the mapping function $\hat{f}_{2}(\cdot), N$ is equal to $50, \gamma$ is 97 , and the outputs of the input pair $\left(a_{i}, S_{2} \cdot b_{i}\right)$ or $\left(S_{1} \cdot a_{i}, b_{i}\right)$ are $\bmod \left(a_{i}+S_{2} \cdot b_{i}, 97\right)$ or $\bmod \left(S_{1} \cdot a_{i}+b_{i}, 97\right)$. Therefore, the maximum output of the $\hat{f}_{2}\left(a_{i}, b_{i}\right)$ is 51 . The reason we choose $\gamma$ to be the maximum prime number in $2 \cdot N$ instead of the maximum prime number in $\max \left(h_{i}+l_{i}\right)$ is that messages can be decoded when $a_{i}>47$ or $b_{i}>47$ without any loss. In addition, the cardinalities of $\hat{f}_{1}\left(a_{i}, b_{i}\right)$ and $\hat{f}_{2}\left(a_{i}, b_{i}\right)$ are greater than the cardinalities of $\mathbf{a}^{T}$ and $\mathbf{b}^{T}$. Thus, for these two mapping functions $\hat{f}_{1}(\cdot)$ and $\hat{f}_{2}(\cdot)$, the system operates in a higher transmission rate in order to fully utilize the available subcarriers.

Once the output symbols $\hat{f}\left(\mathbf{a}^{T}, S_{2} \cdot \mathbf{b}^{T}\right)$ and $\hat{f}\left(S_{1} \cdot \mathbf{a}^{T}, \mathbf{b}^{T}\right)$, are generated, we apply arithmetic code to encode each symbol to the specific codeword using the corresponding codebook, $Q_{x}$ and $Q_{y}$, at each encoder. Cardinalities of these two codebooks used to encode symbols at each encoder are varying with respect to the mapping functions and $N$. For the mapping function $\hat{f}_{1}(\cdot)$, the cardinality of each codebook is 27; however, the cardinality of codebooks for the mapping function $\hat{f}_{2}(\cdot)$ with larger $N$ is 51 . Then, we use a MB-OFDM system with 16-QAM signal mapper/demapper, 4096-point IFFT/FFT $\left(N_{T}=4096\right)$, and $N_{C P}=256$ to transmit the input bit stream over the AWGN channel. Assume the operating bandwidth of the communications system is $20 \mathrm{MHz}$, and each sample is generated by a sampling frequency $5 \mathrm{MHz}$. The raw transmission bit rate of this MB-OFDM system is $18.8 \mathrm{Mbps}$. In addition, the subcarrier spacing, $\Delta f$, is about $4.882 \mathrm{MHz}$.

Some system parameters of the proposed VTR-MBOFDM system are listed in Table 2, where $x[0]$ means $S_{2}=0$ and $\hat{f}\left(\mathbf{a}^{T}, 0\right)$ are encoded, $y[0]$ means $S_{1}=0$ and

\begin{tabular}{|c|c|c|c|}
\hline & \multicolumn{3}{|c|}{ prob, $\hat{f}_{1}(\cdot), N=10$} \\
\hline & $x[0]$ & $y[0]$ & $x[1](y[1])$ \\
\hline Entropy & 3.1036 & 3.1036 & 3.8950 \\
\hline $\begin{array}{l}\text { Encoding rate (bits/sample), } \\
R_{\text {enc }}=R_{\text {enc }, x\left[S_{2}\right]} \text { or } R_{\text {enc,y[0] }}\end{array}$ & 4.5091 & 4.5091 & 5.3636 \\
\hline $\begin{array}{l}\text { Averaged number of used } \\
\text { subcarrier }\left\lceil\frac{L \cdot R_{\text {enc }}}{M}\right\rceil\end{array}$ & 1128 & 1128 & 1341 \\
\hline $\begin{array}{l}\text { Approximately allocated } \\
\text { bandwidth }(\mathrm{MHz})\left[\frac{L \cdot R_{\text {enc }}}{M}\right] \cdot \Delta f\end{array}$ & 5.5 & 5.5 & 6.5 \\
\hline $\begin{array}{l}\text { Transmission bit rate (Mbps), } \\
R_{b}=\left\lceil\frac{L \cdot R_{\text {enc }}}{M}\right\rceil \cdot \frac{f_{s} \cdot M}{N_{T}+N_{C P}}\end{array}$ & 5.18 & 5.18 & 6.16 \\
\hline & moc & $\operatorname{lar}, \hat{f}_{2}(\cdot)$ & $N=50$ \\
\hline & $x[0]$ & $y[0]$ & $x[1](y[1])$ \\
\hline Entropy & 5.3790 & 5.3790 & 6.3344 \\
\hline $\begin{array}{l}\text { Encoding rate (bits/sample), } \\
R_{\text {enc }}=R_{\text {enc }, x\left[S_{2}\right]} \text { or } R_{\text {enc,y[0] }}\end{array}$ & 7.8200 & 7.8200 & 7.8200 \\
\hline $\begin{array}{l}\text { Averaged number of used } \\
\text { subcarrier }\left\lceil\frac{L \cdot R_{\text {enc }}}{M}\right\rceil\end{array}$ & 1955 & 1955 & 1955 \\
\hline $\begin{array}{l}\text { Approximately allocated } \\
\text { bandwidth }(\mathrm{MHz})\left\lceil\frac{L \cdot R_{\text {enc }}}{M}\right\rceil \cdot \Delta f\end{array}$ & 9.5 & 9.5 & 9.5 \\
\hline $\begin{array}{l}\text { Transmission bit rate (Mbps), } \\
R_{b}=\left[\frac{L \cdot R_{\text {enc }}}{M}\right] \cdot \frac{f_{s} \cdot M}{N_{T}+N_{C P}}\end{array}$ & 8.98 & 8.98 & 8.98 \\
\hline
\end{tabular}

Table 2 System parameters of the proposed VTR-MB-OFDM system

$\hat{f}\left(0, \mathbf{b}^{T}\right)$ are encoded, $x[1]$ means $S_{2}=1$ and $\hat{f}\left(\mathbf{a}^{T}, \mathbf{b}^{T}\right)$ are encoded, and the system parameters of $x[1]$ are equal to the system parameters of $y[1]$. In Table 2, the encoding rates are greater than the entropies. It is important that the transmission of the proposed VTR-MB-OFDM system is also admissible. In addition, the used subcarriers, the occupied bandwidths, and the transmission rates are estimated using $L \cdot R_{\text {enc }}$ instead of $X$ and $Y$ for both mapping functions, because the entire packet transmission is a stochastic process which means $X$ and $Y$ are not constants, and $R_{\text {enc }}$ provides the averaged codeword length of possible output data samples. Furthermore, two important issues need to be addressed in Table 2. First, if the mapping function at both encoders is $\hat{f}_{1}(\cdot)$, the system parameters of $x[0]$ are equal to the system parameters of $y[0]$. This is because $\left\{a_{i}\right\}$ and $\left\{b_{i}\right\}$ have the same pdf. Second, the system parameters of $x[0], y[0]$, and $x[1]$ are the same using the mapping function $\hat{f}_{2}(\cdot)$, because $\hat{f}_{2}(\cdot)$ maps the input pair $\left(a_{i}, S_{2} \cdot b_{i}\right)$ or $\left(S_{1} \cdot a_{i}, b_{i}\right)$ to a single value at both encoders. For the mapping function $\hat{f}_{1}(\cdot), 4$ different switch configurations in $\left[S_{1} S_{2}\right]$ generate different transmission rates to transmit correlated sequences $\left\{a_{i}\right\}$ and $\left\{b_{i}\right\}$. Moreover, the rate factors of the proposed VTR-MBOFDM system are approximately equal to $0.55,0.6,0.66$, and 0.96 . 
The performance of the proposed VTR-MB-OFDM system over the noiseless channel is shown in Figure 5. We evaluate the effects of 16 different switch configurations to the system performance using two different mapping functions, $\hat{f}_{1}(\cdot)$ and $\hat{f}_{2}(\cdot)$, at both encoders in Figure $5 \mathrm{a}, \mathrm{b}$, respectively. If the mapping function $\hat{f}_{1}(\cdot)$ is chosen, the SER reaches to 0.5 or 1 when

$$
\left\{\begin{array}{l}
{\left[\begin{array}{ll}
S_{1} S_{2}
\end{array}\right] \neq\left[\begin{array}{ll}
0 & 0
\end{array}\right]} \\
{\left[\begin{array}{ll}
S_{3} S_{4}
\end{array}\right] \neq\left[\begin{array}{ll}
1 & 1
\end{array}\right]} \\
{\left[\begin{array}{ll}
S_{3} S_{4}
\end{array}\right] \neq\left[\begin{array}{ll}
S_{1} & S_{2}
\end{array}\right] .}
\end{array}\right.
$$

Moreover, if the mapping function $\hat{f}_{2}(\cdot)$ is chosen, the SER reaches to 0.5 or 1 when

$$
\left[S_{1} S_{2}\right]=\left[\begin{array}{ll}
1 & 1
\end{array}\right]
$$

or

$$
\left\{\begin{array}{l}
{\left[\begin{array}{ll}
S_{1} S_{2} &
\end{array} \neq\left[\begin{array}{ll}
0 & 0
\end{array}\right]\right.} \\
{\left[\begin{array}{l}
S_{3} S_{4} \\
S_{3}
\end{array}\right]\left[\begin{array}{ll}
1 & 1
\end{array}\right]} \\
{\left[S_{3} S_{4}\right] \neq\left[\begin{array}{ll}
S_{1} & S_{2}
\end{array}\right] .}
\end{array}\right.
$$

For $\left[\begin{array}{ll}S_{1} & S_{2}\end{array}\right]=\left[\begin{array}{ll}0 & 0\end{array}\right]$, the input pairs are mapped independently. Thus, decoders can decode the estimated bit stream correctly with or without the side information. In

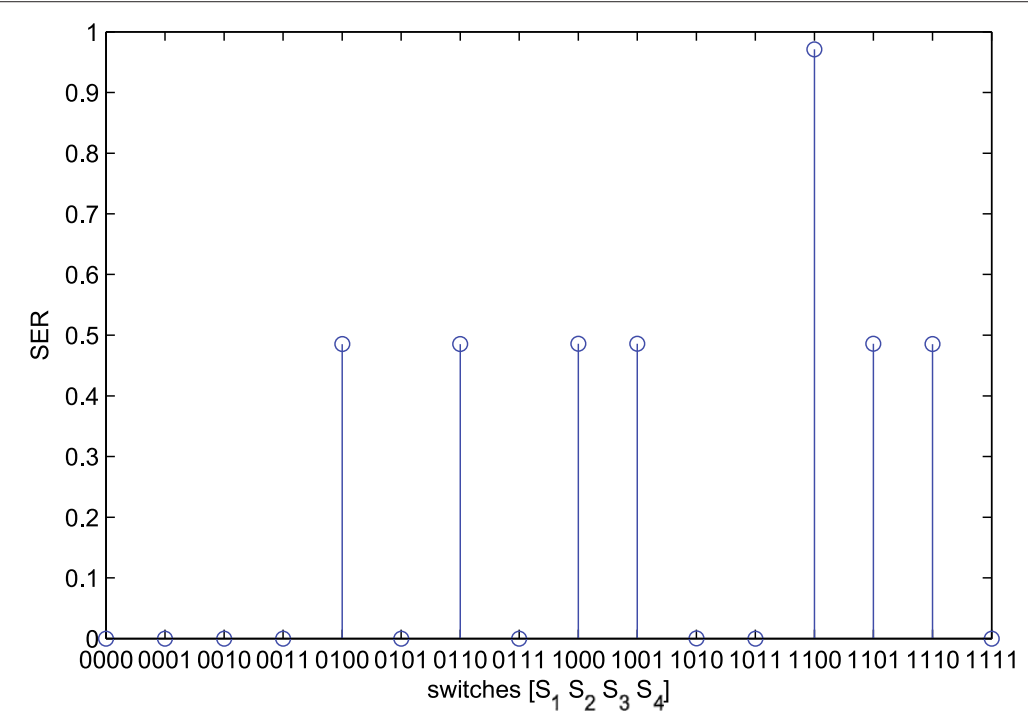

(a)

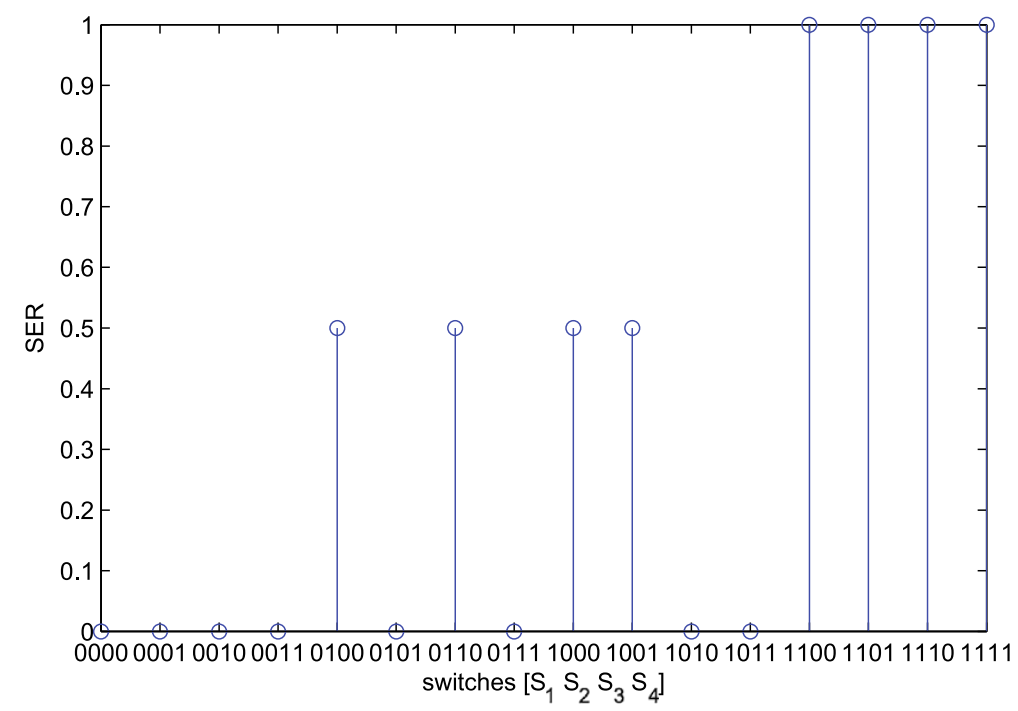

(b)

Figure 5 The performance of the proposed VTR-MB-OFDM system in the noiseless channel using two different mapping functions.

(a) $\hat{f}_{1}(\cdot)$ with switch configurations $\left[S_{1} S_{2} S_{3} S_{4}\right]=[x x x x]$. (b) $\hat{f}_{2}(\cdot)$ with switch configurations $\left[S_{1} S_{2} S_{3} S_{4}\right]=[x x x x]$. ' $x^{\prime}$ means ' 0 ' or ' 1 '. Therefore, 16 different switch configurations $\left[S_{1} S_{2} S_{3} S_{4}\right]$ are considered. 


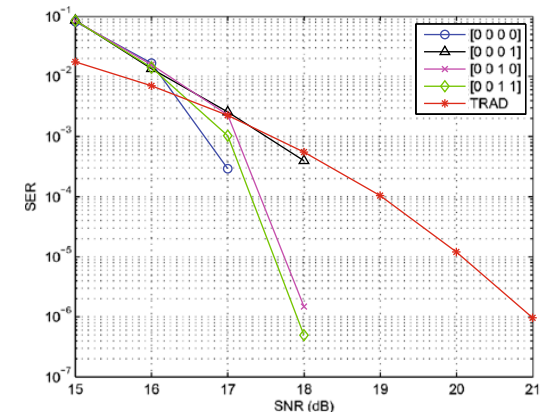

(a)

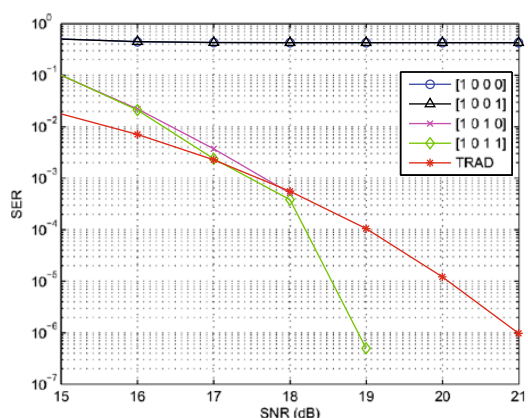

(c)

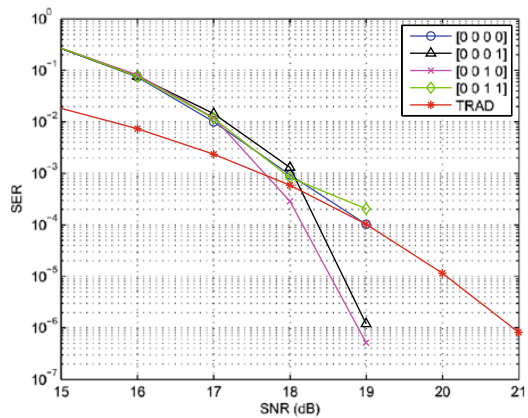

(e)

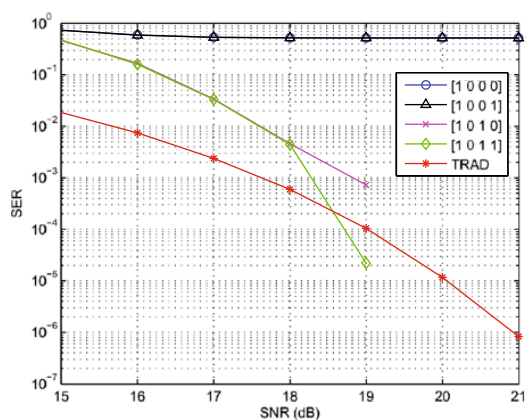

(g)

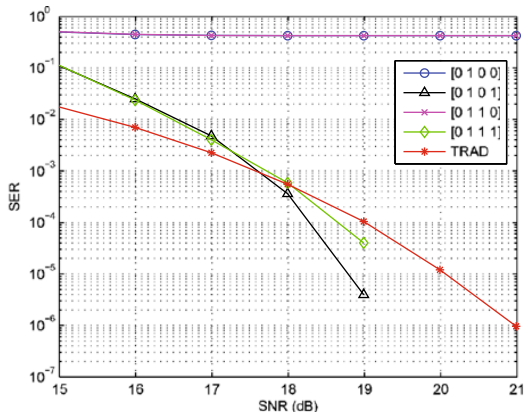

(b)

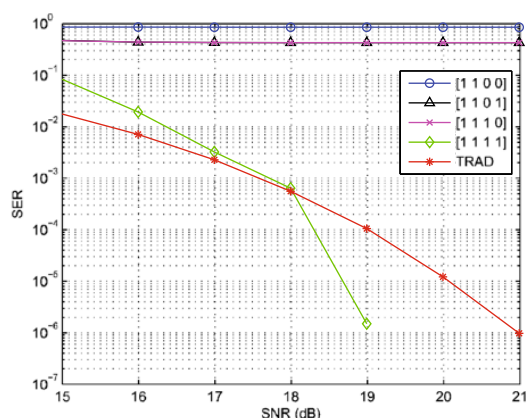

(d)

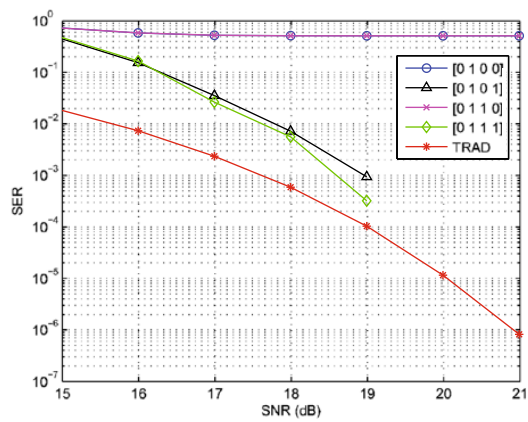

(f)

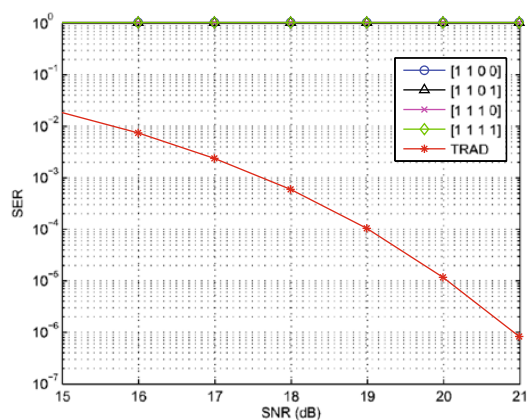

(h)

Figure 6 The performance of the proposed VTR-MB-OFDM communications system in the AWGN channel using two different mapping

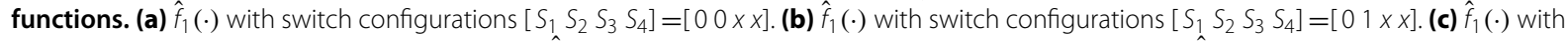
switch configurations $\left[S_{1} S_{2} S_{3} S_{4}\right]=\left[\begin{array}{lll}1 & 0 & x\end{array}\right]$. (d) $\hat{f}_{1}(\cdot)$ with switch configurations $\left[S_{1} S_{2} S_{3} S_{4}\right]=\left[\begin{array}{lll}1 & 1 x & x\end{array}\right]$. (e) $\hat{f}_{2}(\cdot)$ with switch configurations

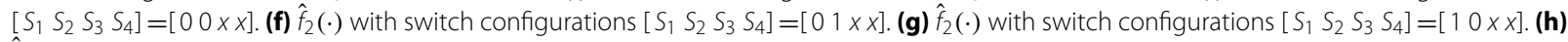
$\hat{f}_{2}(\cdot)$ with switch configurations $\left[S_{1} S_{2} S_{3} S_{4}\right]=\left[\begin{array}{lll}1 & 1 & x\end{array}\right]$. ' $x$ ' means ' $O^{\prime}$ or ' 1 '. From Figure $6 a-d$, effects caused by 16 different switch configurations to the system performance using the mapping function $\hat{f}_{1}(\cdot)$ are evaluated, and then we also evaluate the effects caused by 16 different switch configurations using the mapping function $\hat{f}_{2}(\cdot)$ to the system performance in Figure $6 \mathrm{e}-\mathrm{h}$. 
the case of [ $\left.S_{3} S_{4}\right] \neq\left[S_{1} S_{2}\right]$ for both mapping functions, decoders decode the estimated bit stream in a way different from the way that encoders generate these encoded bit stream. In addition, for the mapping function $\hat{f}_{2}(\cdot)$, the reason we have the highest SER when $\left[S_{1} S_{2}\right]=\left[\begin{array}{ll}1 & 1\end{array}\right]$ is that the decoder can not map a single value back to the original input pair $\left\{\hat{a}_{i}, \hat{b}_{i}\right\}$ with or without the help of the side information.

We demonstrate the performance of the proposed VTRMB-OFDM system over the AWGN channel using two different mapping functions in Figure 6. The system performance using the mapping function $\hat{f}_{1}(\cdot)$ is demonstrated in Figure 6a-d, and then the system performance using the mapping function $\hat{f}_{2}(\cdot)$ is also demonstrated in Figure $6 \mathrm{e}-\mathrm{h}$. From Figure 6 , the system performance over the noiseless channel is the same as the system performance over the noisy channel when SNR is greater than $19 \mathrm{~dB}$. Compared with the traditional uncoded OFDM system, the proposed VTR-MB-OFDM system has at least 1 to $4 \mathrm{~dB}$ gain in SNR to achieve the same SER. In addition, the proposed VTR-MB-OFDM system achieves zero SER for some switch configurations when SNR is greater than $19 \mathrm{~dB}$.

\section{Conclusion}

In this article, two variable transmission rate OFDM systems using network coding schemes are proposed, and the performance of these two proposed systems is evaluated. For the proposed VTR-SISO-OFDM system, the transmission rate is adjusted by selecting the bit stream from the output at different stages with different mapping functions in the encoder; however, how an appropriate mapping function is chosen is important for the system performance. In addition, the cardinalities of the side information also affect the system performance. The larger the codebook size, the less gain in SNR to achieve the same performance as the uncoded communications system. Thus, a trade-off between higher transmission rate and better system performance is necessary. As for the proposed VTR-MB-OFDM system, correlated sources are simultaneously transmitted using different transmission rates. Different switch configurations generate different transmission rates at the transmitter and different system performance at the receiver. Compared with the traditional uncoded OFDM system, simulation results show the proposed VTR-SISO-OFDM system has at least 1 to $3 \mathrm{~dB}$ gain in SNR to achieve the same SER, and the proposed VTR-MB-OFDM system has at least 1 to $4 \mathrm{~dB}$ gain in SNR to achieve the same SER. In addition, for some conditions, zero SER is achievable for both proposed systems when SNR is greater than $19 \mathrm{~dB}$. Moreover, the rate factors of these two proposed system vary from 0.55 to 0.96 .
Competing interests

The authors declare that they have no competing interests.

\section{Acknowledgements}

The authors would like to thank Emeritus Professor John C. Kieffer and anonymous reviewers for their valuable advice which substantially improved this article.

Received: 20 April 2012 Accepted: 17 January 2013

Published: 1 February 2013

References

1. J Proakis, Digital Communications. (McGraw Hill, New York, NY, 2000)

2. G Stuber, Principle of mobile communication. (Kluwer Academic Publishers, Norwell, MA, 2000)

3. Q Zhao, Network source coding: theory and code design for broadcast and multiple access network. Ph.D. thesis, California Institute of Technology (2003)

4. STung, Multiterminal source coding. Ph.D. thesis, Cornell University (1978)

5. C Shannon, A mathematical theory of communication. Bell Systs. Tech. J. 27, 379-423, 623-656 (1948)

6. M Effros, Network source coding: a perspective. IEEE Inf. Theory Soc. Newslett. 57(4), 15-23 (2007)

7. D Slepian, J Wolf, Noiseless coding of correlated information sources. IEEE Trans. Inf. Theory. IT-19(4), 471-480 (1973)

8. H Yamamoto, Wyner-ziv theory for a general function of the correlated sources. IEEE Trans. Inf. Theory. IT-28(5), 1788-1791 (1982)

9. H Feng, M Effros, S Savari, in Proceedings of the Allerton Conference on Communication, Control, and Computing. Functional source coding for networks with receiver side information, (Monticello, IL, 2004), pp. 1419-1427

10. A Orlitsky, J Roche, Coding for computing. IEEE Trans. Inf. Theory. 47(3), 903-917 (2001)

11. R Ahlswedw, N Cai, SY Li, R Yeung, Network information flow. IEEE Trans Inf. Theory. IT-46(4), 1204-1216 (2000)

12. THo, M Médard, R Koetter, D Karger, M Effros, J Shi, B Leong, A random linear network coding approach to multicast. IEEE Trans. Inf. Theory. 52(10), 4413-4430 (2006)

13. R Nee, R Prasad, OFDM Wireless Multimedia Communication. (Artech House, Norwood, MA, 2000)

14. IEEE 802.15 Task Group, Multi-band OFDM Physical Layer Proposal for IEEE 802.15 Task Group 3a (2003)

15. J Balakrishnan, A Batra, A Dabak, in Proceedings of IEEE Conference Ultra Wideband System Technology. A multi-band OFDM system for UWB communication, (Virginia, USA, 2003), pp. 354-358

16. R Zhang, L Hanzo, Multiple-source cooperation: from code-division multiplexing to variable-rate network coding. IEEE Trans. Veh. Technol. 60(3), 1005-1015 (2011)

17. A Wyner, J Ziv, The rate-distortion function for source coding with side information at the decoder. IEEE Trans. Inf. Theory. IT-22(1), 1-10 (1976)

18. T Berger, Multiterminal Source Coding. (Springer-verlag, New York, 1978)

19. J Kieffer, A method for proving multiterminal source coding theorems. IEEE Trans. Inf. Theory. IT-27(5), 565-570 (1981)

doi:10.1186/1687-6180-2013-12

Cite this article as: Kung and Parhi: Performance evaluation of variable transmission rate OFDM systems via network source coding. EURASIP Journal on Advances in Signal Processing 2013 2013:12. 